\title{
A theoretical and experimental study of pressure broadening of the oxygen A-band by helium
}

\author{
Dennis L. A. G. Grimminck, ${ }^{1}$ Frans R. Spiering, ${ }^{1}$ Liesbeth M. C. Janssen,,${ }^{1, *}$ \\ Ad van der Avoird, ${ }^{1}$ Wim J. van der Zande, ${ }^{1}$ and Gerrit C. Groenenboom ${ }^{1, \dagger}$ \\ ${ }^{1}$ Institute for Molecules and Materials, \\ Radboud University Nijmegen, Heyendaalseweg 135, \\ 6525 AJ Nijmegen, The Netherlands
}

(Dated: May 6, 2014)

\begin{abstract}
The rotationally resolved magnetic dipole absorption spectrum of the oxygen A-band $b^{1} \Sigma_{g}^{+}(\mathrm{v}=0) \leftarrow X^{3} \Sigma_{g}^{-}(\mathrm{v}=0)$ perturbed by collisions with helium was studied theoretically using the impact approximation. To calculate the relaxation matrix, scattering calculations were performed on a newly computed helium-oxygen $\left(b^{1} \Sigma_{g}^{+}\right)$interaction potential as well as on a heliumoxygen $\left(X^{3} \Sigma_{g}^{-}\right)$interaction potential from the literature. The calculated integrated line cross sections and broadening coefficients are in good agreement with experimental results from the literature. Additionally, cavity ring-down experiments were performed in the wings of the spectral lines for a quantitative study of line-mixing, i.e., the redistribution of rotational line intensities by helium-oxygen collisions. It is shown that inclusion of line-mixing in the theory is required to reproduce the experimentally determined absolute absorption strengths as a function of the density of the helium gas.
\end{abstract}

\footnotetext{
*Present address: Department of Chemistry, Columbia University, 3000 Broadway, New York, New York 10027, USA

${ }^{\dagger}$ Electronic mail: Gerritg@theochem.ru.nl
} 


\section{INTRODUCTION}

The near-infrared $(\approx 760 \mathrm{~nm})$ A-band extinction of molecular oxygen $\left(\mathrm{O}_{2}\right)$ is of increasing atmospheric importance. It is used extensively for the calibration of satellite instruments that help to determine atmospheric temperature and pressure $[1,2]$. Due to its high molecular symmetry and lack of an electric dipole moment, $\mathrm{O}_{2}$ is a poor absorber with only weakly allowed transitions in the infrared. However, the high atmospheric concentration of molecular oxygen $(\approx 20 \%)$ and the long optical path lengths that can be obtained in the Earth's atmosphere lead to an almost saturated $\mathrm{O}_{2}$ infrared spectrum. As a consequence, small variations in e.g. the Earth's oxygen content become noticeable only by changes in the low-intensity wings of the spectral lines.

The extinction of light by atmospheric oxygen in the A-band region is understood to originate from (i) Rayleigh scattering, the elastic scattering of light on subwavelength particles (ii) collision induced absorption (CIA) [3-5], the absorption of light by a transient electric dipole moment, and (iii) magnetic dipole absorption [6]. Much effort has been invested to quantify the contribution of these mechanisms experimentally [7-9]. Figure 1 shows a construction of the pure oxygen A-band spectrum based on a sum of these different contributions. The strongest absorption features in the A-band region are due to the electronic magnetic dipole transition from the ground state $X^{3} \Sigma_{g}^{-}(\mathrm{v}=0, J)$ to the excited state $b^{1} \Sigma_{g}^{+}\left(\mathrm{v}=0, J^{\prime}\right)$, in which the rotational quantum number $J$ changes by either $\Delta J=J^{\prime}-J=-1$ ( $P$ branch) or $\Delta J=+1$ ( $R$ branch) [6]. Although this transition violates the selection rules for magnetic dipole absorption [10] — the spin multiplicity ${ }^{1} \Sigma \leftarrow{ }^{3} \Sigma$ and the reflection symmetry $\Sigma^{+} \leftarrow \Sigma^{-}$are not conserved- spin-orbit coupling makes the transition allowed [11]. We note that the latter does not occur for the electric dipole transition - forbidden due to the change in spin multiplicity and the conservation of parity $\Sigma_{g} \leftarrow \Sigma_{g}$. An electric quadrupole transition is also not the cause of the radiation absorption, since $O(\Delta J=-2)$ and $S(\Delta J=+2)$ branches with almost equal intensity as the $P$ and $R$ branches should then be visible in the experimental spectrum [10]. As can be seen in Fig. 1, Rayleigh scattering and CIA contribute broad and relatively weak absorption features in the A-band region. A minor, but not unimportant feature of the spectrum concerns the regions between the peaks of the magnetic dipole absorption contribution. The absorption in these regions is determined by the spectral wings, which are affected by line-mixing [12, 13], 
i.e., the redistribution of rotational spectral line intensities by collisions with another gas. Although this effect is negligible on the scale of Fig. 1, it can serve as a useful probe in experiments which are prevented by saturation effects in the regions of stronger absorption. Moreover, line-mixing provides a sensitive test for quantitative theoretical models of oxygen absorption spectroscopy.

To our knowledge, there is no first principles prediction in the literature of an electronic magnetic dipole absorption spectrum of molecular oxygen influenced by collisions with a foreign gas. In a broader perspective, there is no fully quantum mechanical treatment available in the literature that treats an electronic transition of a molecule perturbed by collisions. The reason could be the requirement for an accurate interaction potential of the perturbing particle with the electronically excited state of the absorbing molecule.

These considerations motivated our choice to study, both theoretically and experimentally, the collisional effects of helium (He) atoms on the A-band magnetic dipole absorption spectrum of molecular oxygen. More explicitly, we focus on the spectral lines arising from the $b^{1} \Sigma_{g}^{+}(\mathrm{v}=0) \leftarrow X^{3} \Sigma_{g}^{-}(\mathrm{v}=0)$ transition in ${ }^{16} \mathrm{O}_{2}$, and we study the coupling between these lines, i.e., the line-mixing, due to inelastic He- $\mathrm{O}_{2}$ collisions. Rayleigh scattering and CIA are not taken into account in our theoretical model, and experimentally we also separate these contributions from the spectrum. The impact approximation is used for the theoretical description of the dynamics of the molecular system. In this approximation, strong collisions, which change the absorber's wavefunction significantly, are assumed to be well separated in time [14]. Quantum mechanical scattering calculations that capture the $\mathrm{He}-\mathrm{O}_{2}$ collision dynamics, and thus ultimately determine the pressure-broadening and line-mixing effects, are performed on interaction potentials for helium with the $X$ and $b$ state of molecular oxygen. A new interaction potential for the $b$ state was calculated for this work, and for the $X$ state a potential was taken from the literature [15]. The prediction of line-mixing provides a sensitive test for our calculated results. To test the predictions, cavity ring-down experiments were carried out to measure the absolute absorption intensities for this system as a function of pressure, with a sensitivity of about $10^{-7} \mathrm{~cm}^{-1}$. We used the experimental data for a quantitative comparison between theory and experiment regarding the shape of the lines relatively far from the line center, where line-mixing is most pronounced. These results show the importance of including line-mixing in the theory to describe the absorption spectrum correctly. 
In the following sections of this paper, we start with a review of the theory of the rotationally resolved spectroscopy of the oxygen A-band. The expression for the absorption spectrum in the impact approximation will be given. This is followed by the description of the calculation of the interaction potential for the helium atom with the oxygen $b$ state, and the performed scattering calculations. Then the cavity ring-down experiments will be described, including the processing of the data to remove the contribution of Rayleigh scattering and CIA. In the results section, we first show the comparison between theoretical and experimental integrated line cross sections and pressure broadening coefficients. These quantities are relatively insensitive to the effects of line-mixing, and hence line-mixing is neglected in these calculations. Next, we compare our theory with rigorous inclusion of line-mixing effects to our measured cavity ring-down data for absolute absorption strengths in the "valleys" between spectral lines. Finally, we compare our first principles predictions with a successful semi-empirical model by Tonkov et al. $[12,13]$, which also accounts for linemixing, and find good quantitative agreement. Based on these comparisons, we conclude that our full quantum mechanical framework leads to good quantitative agreement when line-mixing effects are included.

\section{THEORY}

\section{A. Rotational structure of the $X^{3} \Sigma_{g}^{-}$and $b^{1} \Sigma_{g}^{+}$states of $\mathbf{O}_{2}$}

In this section we focus on the rotational and electronic structure of the $X$ and $b$ states. We assume that both the $X$ and $b$ state are in the vibrational ground state $(\mathrm{v}=0)$, and hence the vibrational part of the wavefunction will not be explicitly considered in the theory. The electronic ground state of molecular oxygen is an open shell state with term symbol $X^{3} \Sigma_{g}^{-}$. The relatively weak coupling between nuclear rotation and electronic spin - see the value of $\lambda_{N S}$ in Table I - warrants the expansion of the ground state fine structure states $\left|F_{i} J M_{J} ; X^{3} \Sigma_{g}^{-}\right\rangle$in a Hund's case (b) basis

$$
\begin{array}{r}
\left|F_{i} J M_{J} ; X^{3} \Sigma_{g}^{-}\right\rangle=\sum_{N=J-1}^{J+1} a_{N}^{F_{i}} \sum_{M_{N}, M_{S}} \sqrt{\frac{2 N+1}{4 \pi}} D_{M_{N}, 0}^{N}(\alpha, \beta, 0)^{*} \\
\times\left|X^{3} \Sigma_{g}^{-}, S, M_{S}\right\rangle\left\langle N M_{N} S M_{S} \mid J M_{J}\right\rangle
\end{array}
$$


where $N$ and $S$ are the rotational and spin angular momentum quantum numbers with laboratory-axis projection quantum numbers $M_{N}$ and $M_{S}$, respectively, $J$ is the total angular momentum arising from coupling of $N$ and $S$, and $M_{J}$ is its laboratory-axis projection. For the $X^{3} \Sigma_{g}^{-}$state we have $S=1$. The two-angle normalized Wigner $D$-functions $\sqrt{(2 N+1) / 4 \pi} D_{M_{N}, 0}^{N}(\alpha, \beta, 0)^{*}$, which are nuclear rotation eigenfunctions, are coupled to the electronic spin by the Clebsch-Gordan coefficient $\left\langle N M_{N} S M_{S} \mid J M_{J}\right\rangle$ and mixed by the expansion coefficients $a_{N}^{F_{i}}$. Here $\alpha$ and $\beta$ are azimuthal and zenith angles of the $\mathrm{O}_{2}$ axis, respectively.

The fine structure label $F_{i}$ has three possible values: $F_{1}, F_{2}$, and $F_{3}$. According to the convention used in [16] the expansion coefficients $a_{N}^{F_{i}}$ in Eq. (1) are of the form

$$
\begin{aligned}
& a_{N=J-1}^{F_{1}}=a_{N=J+1}^{F_{3}}=\cos \phi \\
& a_{N=J}^{F_{2}}=1 \\
& a_{N=J+1}^{F_{1}}=-a_{N=J-1}^{F_{3}}=\sin \phi
\end{aligned}
$$

and they follow from diagonalization of the molecular Hamiltonian [17]. Equation (2) shows that the $F_{1}$ and $F_{3}$ states are constructed from $N=J-1$ and $N=J+1$ rotational states. The mixing-angle $\phi$ decreases if states of higher $N$ are involved.

The $X^{3} \Sigma_{g}^{-}$state of ${ }^{16} \mathrm{O}_{2}$ has only odd values of $N$ because the Pauli principle requires the total wave function to be even under permutation of the identical bosonic nuclei. For the nuclear wave function the permutation operator is equal to inversion and the parity of the nuclear wave function is $(-1)^{N}$. The permutation operator also acts on the electronic wave function, because it rotates the frame over $180^{\circ}$ around an axis perpendicular to the diatomic axis. This rotation can be written as the product of an inversion of the electronic coordinates and a reflection in a plane containing the diatomic axis. Since the ground state is gerade the inversion is even, but for a $\Sigma^{-}$state the reflection is odd. So for odd $N$ the total wave function is even under the permutation operator.

For the excited $b^{1} \Sigma_{g}^{+}$state of ${ }^{16} \mathrm{O}_{2}$, which has $S=0$, we can write

$$
\left|N^{\prime} M_{N}^{\prime} ; b^{1} \Sigma_{g}^{+}\right\rangle=\sqrt{\frac{2 N^{\prime}+1}{4 \pi}} D_{M_{N}^{\prime}, 0}^{N^{\prime}}(\alpha, \beta, 0)^{*}\left|b^{1} \Sigma_{g}^{+}\right\rangle .
$$

Primes are used here to distinguish between the rotational quantum numbers of the $b$ state from those of the $X$ state. Following a similar reasoning as above, it can be shown that $N^{\prime}$ has only even values. 


\section{B. Magnetic transition dipole matrix elements}

The rovibronic transitions between the states $\left|F_{i} J M_{J} ; X^{3} \Sigma_{g}^{-}\right\rangle$and $\left|N^{\prime} M_{N}^{\prime} ; b^{1} \Sigma_{g}^{+}\right\rangle$(both vibrational ground states) have magnetic dipole character although they do not obey the usual selection rules [10], since the spin multiplicity ${ }^{1} \Sigma \leftarrow{ }^{3} \Sigma$ and the reflection symmetry $\Sigma^{+} \leftarrow \Sigma^{-}$are not conserved. The mechanism responsible for the non-zero transition probability is spin-orbit coupling [11]. Neither electric dipole nor quadrupole transitions contribute to the spectrum as discussed in the introduction.

To explain the magnetic dipole transition mechanism we first expand the oxygen ground state wave function from Eq. (1) in a Hund's case (a) basis

$$
\begin{gathered}
\left|F_{i} J M_{J} ; X^{3} \Sigma_{g}^{-}\right\rangle=\sum_{N=J-1}^{J+1} a_{N}^{F_{i}} \sum_{\Omega} \sqrt{\frac{2 N+1}{4 \pi}} D_{M_{J}, \Omega}^{J}(\alpha, \beta, 0)^{*} \\
\times\left|X^{3} \Sigma_{g, \Omega}^{-}, S, \Omega\right\rangle\langle N 0 S \Omega \mid J \Omega\rangle,
\end{gathered}
$$

for which we used

$$
\left|X^{3} \Sigma_{g}^{-}, S M_{S}\right\rangle=\sum_{\Omega}\left|X^{3} \Sigma_{g}^{-}, S \Omega\right\rangle D_{M_{S}, \Omega}^{S}(\alpha, \beta, 0)^{*},
$$

and the standard expression for a product of two Wigner $D$-functions [18]. The quantum number $\Omega$ is the projection of the total angular momentum $J$ on the molecular axis, which equals the spin projection for a $\Sigma$ state.

The electronic magnetic dipole operator is given by

$$
\hat{\boldsymbol{\mu}}=\sum_{i} \frac{\mu_{B}}{\hbar}\left(\hat{\boldsymbol{l}}_{i}+g_{e} \hat{\boldsymbol{s}}_{i}\right) \equiv \frac{\mu_{B}}{\hbar}\left(\hat{\boldsymbol{L}}+g_{e} \hat{\boldsymbol{S}}\right)
$$

with $\hat{\boldsymbol{l}}_{i}$ and $\hat{\boldsymbol{s}}_{i}$ being the orbital and spin angular momentum vector operators of electron $i, \mu_{B}$ the Bohr magneton, $g_{e} \approx 2.0$ the electron g-factor, and $\hbar$ Planck's reduced constant. Absorption intensities are directly proportional to the square of the space-fixed (SF) transition dipole moments, which in turn are related to the body-fixed (BF) dipole operator via the transformation

$$
\hat{\mu}_{m}^{S F}=\sum_{k} \hat{\mu}_{k}^{B F} D_{m, k}^{1}(\alpha, \beta, 0)^{*} .
$$

The spherical components $\hat{\mu}_{m}$ and $\hat{\mu}_{k}$ of the SF and BF dipole moment operators are related to the Cartesian components as

$$
\begin{aligned}
\hat{\mu}_{0} & =\hat{\mu}_{z} \\
\hat{\mu}_{ \pm 1} & =\mp\left(\hat{\mu}_{x} \pm i \hat{\mu}_{y}\right) / \sqrt{2} .
\end{aligned}
$$


The combination of Eqs. (3), (4), and (7) leads to an expression for the transition dipole matrix elements

$$
\begin{aligned}
& \left\langle F_{i} J M_{J} ; X^{3} \Sigma_{g}^{-}\left|\hat{\mu}_{m}^{S F}\right| N^{\prime} M_{N}^{\prime} ; b^{1} \Sigma_{g}^{+}\right\rangle= \\
& (-1)^{J-M_{J}} \sqrt{2 J+1}\left(\begin{array}{ccc}
J & 1 & N^{\prime} \\
-M_{J} & m & M_{N}^{\prime}
\end{array}\right)\left\langle F_{i} J ; X^{3} \Sigma_{g}^{-} \| \mu|| N^{\prime} ; b^{1} \Sigma_{g}^{+}\right\rangle,
\end{aligned}
$$

where the factor in large brackets denotes a Wigner three- $j$ symbol and the Wigner-Eckart theorem reduced matrix element is given by

$$
\begin{aligned}
& \left\langle F_{i} J ; X^{3} \Sigma_{g}^{-}\|\mu\| N^{\prime} ; b^{1} \Sigma_{g}^{+}\right\rangle=\sum_{N, \Omega} a_{N}^{F_{i}{ }^{*}}(-1)^{N-J-1} \sqrt{(2 N+1)\left(2 N^{\prime}+1\right)} \\
& \quad \times\left(\begin{array}{ccc}
J & 1 & N^{\prime} \\
-\Omega & \Omega & 0
\end{array}\right)\left(\begin{array}{ccc}
N & 1 & J \\
0 & \Omega & -\Omega
\end{array}\right)\left\langle X^{3} \Sigma_{g, \Omega}^{-}\left|\hat{\mu}_{\Omega}^{B F}\right| b^{1} \Sigma_{g}^{+}\right\rangle .
\end{aligned}
$$

The integrated line cross sections [19] that can be compared to experimental results can be written as

$$
I_{F_{i}, J, N^{\prime}}=\frac{8 \pi^{3} \tilde{\nu}_{F_{i}, J, N^{\prime}}}{\left(4 \pi \varepsilon_{0}\right) 3 h c^{3} Z} e^{-h c \tilde{\nu}_{F_{i}, J}^{(X)} \bar{\beta}}(2 J+1)\left|\left\langle F_{i}, J ; X^{3} \Sigma_{g}^{-}\|\mu\| N^{\prime} ; b^{1} \Sigma_{g}^{+}\right\rangle\right|^{2} .
$$

Here $I_{F_{i}, J, N^{\prime}}$ is in unit length per molecule, $\tilde{\nu}_{F_{i}, J, N^{\prime}}$ is the wavenumber of the transition, $h$ Planck's constant, $c$ the speed of light, $\varepsilon_{0}$ the vacuum permittivity constant, $Z$ the partition sum of molecular oxygen [20], $h c \tilde{\nu}_{F_{i}, J}^{(X)}$ the energy of state $\left|F_{i} J ; X^{3} \Sigma_{g}^{-}\right\rangle$, and $\bar{\beta}=1 / k_{B} T$, with $k_{B}$ the Boltzmann constant, and $T$ the absolute temperature.

The final step is the calculation of the body-fixed electronic matrix element $\left\langle X^{3} \Sigma_{g, \Omega}^{-}\left|\hat{\mu}_{\Omega}^{B F}\right| b^{1} \Sigma_{g}^{+}\right\rangle$, which is extensively treated in Refs. 21 and 11 . The contribution from the orbital angular momentum term $\hat{\boldsymbol{L}}$ in the magnetic dipole operator, Eq. (6), is about 60 times smaller than that of the spin term $g_{e} \hat{\boldsymbol{S}}$ [11], so we only describe the evaluation of the latter. The magnetic dipole transition borrows its main intensity from the admixture of the $\left|X^{3} \Sigma_{g}^{-}\right\rangle$ground state component with $\Omega=0$ directly into the $b$ state, with a mixing coefficient

$$
C=\frac{\left\langle{ }^{3} \Sigma_{g, \Omega=0}^{-}\left|\hat{H}_{S O}\right| b^{1} \Sigma_{g}^{+}\right\rangle}{E_{b}-E_{X}}
$$

where $E_{X}$ and $E_{b}$ are the energies of the ground and excited $b$ state, respectively, and $\hat{H}_{S O}$ is the spin-orbit operator responsible for the mixing. The only resulting non-zero matrix elements for $\Omega= \pm 1$ are given by

$$
\left\langle X^{3} \Sigma_{g, \pm 1}^{-}\left|\hat{\mu}_{ \pm 1}^{B F}\right| b^{1} \Sigma_{g}^{+}\right\rangle=\frac{g_{e} \mu_{B}}{\hbar}\left\langle X^{3} \Sigma_{g, \pm 1}^{-}\left|\frac{\mp\left(\hat{S}_{x} \pm i \hat{S}_{y}\right)}{\sqrt{2}}\right| X^{3} \Sigma_{g, 0}^{-}\right\rangle C=\mp g_{e} \mu_{B} C .
$$


Note that $\hat{S}_{x} \pm i \hat{S}_{y}$ are ladder operators which, for a $\Sigma$ state, act directly on the $\Omega$ projection quantum numbers.

The mixing coefficient $C$ in Eq. (11), including averaging over the $\mathrm{O}_{2}$ vibrational coordinate $r$ but without considering the rotations that we treat in the present paper, was calculated $a b$ initio by Minaev et al. [11]. Also the much smaller contribution from the $\hat{\boldsymbol{L}}$ term in the magnetic dipole operator of Eq. (6) was calculated. Different values for the total transition moment $g_{e} \mu_{B} C$ are given in Ref. 11. The value of $0.0268 \mu_{B}$ was obtained by Klotz et al. [21] from an ab initio calculation of the numerator in Eq. (11) at the ground state equilibrium $\mathrm{O}-\mathrm{O}$ distance $r_{e}=2.28 a_{0}$ and the experimental value of the excitation energy $E_{b}-E_{X}$ in the denominator. The value of $0.0241 \mu_{B}$ is the best value of Ref. 11 obtained from a full ab initio calculation of both the numerator and the denominator of the expression in Eq. (11) as a function of the O-O distance $r$ and averaging over the vibrational ground states of the electronic ground and $b$ states. Computation of the excitation energies in the denominator from empirical RKR (Rydberg-Klein-Rees) potentials for the ground and $b$ states instead of using the $a b$ initio values yields practically the same value $g_{e} \mu_{B} C=0.0268 \mu_{B}$ as that obtained somewhat fortuitously by Klotz et al.. This is considered as the best value, so we used it in our calculations of the integrated line cross sections. Minaev et al. also report measured radiative life times, some of which are close to the life time that corresponds to this best value of the transition moment.

\section{Spectrum in the impact approximation}

The rotationally resolved A-band magnetic dipole absorption spectrum concerns transitions of the type $\left|N^{\prime} ; b^{1} \Sigma_{g}^{+}\right\rangle \leftarrow\left|F_{i}, J ; X^{3} \Sigma_{g}^{-}\right\rangle$, both in the vibrational ground state. In the impact approximation it is assumed that strong collisions, which change the absorber's wavefunction significantly, are well separated in time[14]. This approximation is combined with the assumption that the internal states of the perturbing particles are not affected by their interactions, and that the absorbers experience isotropic gas surroundings. Collisional effects on the spectrum are described in terms of binary collisions of absorber and perturber. Note that the influence of the motion of the center of mass of this binary collision complex on the absorption of light is neglected, therefore the theory does not predict the Doppler or Dicke effect. In the impact approximation, the collision dynamics is captured by elastic and 
inelastic scattering amplitudes. This leads to the expression for the magnetic dipole absorption spectrum in unit length squared per molecule at the spectral position with wavenumber $\tilde{\nu}[19,22-25]$

$$
\begin{gathered}
F(\tilde{\nu})=\frac{1}{\pi} \frac{8 \pi^{3} \tilde{\nu}}{\left(4 \pi \varepsilon_{0}\right) 3 h c^{3}} \operatorname{Im}\left[\sum_{F_{i}^{\prime}, J^{\prime}, N^{\prime} ; F_{i}, J, N}\left\langle F_{i}^{\prime}, J^{\prime} ;{ }^{3} \Sigma_{g}^{-}\|\mu\| N^{\prime} ;{ }^{1} \Sigma_{g}^{+}\right\rangle^{*}\right. \\
\left.\left[\mathbf{A}^{-1}\right]_{F_{i}^{\prime}, J^{\prime}, N^{\prime} ; F_{i}, J, N} P_{F_{i}, J}\left\langle F_{i}, J ;{ }^{3} \Sigma_{g}^{-}\|\mu\| N ;{ }^{1} \Sigma_{g}^{+}\right\rangle\right] .
\end{gathered}
$$

Here $\operatorname{Im}[. .$.$] means the imaginary part of the expression in the brackets, see Eq. (10) for$ further explanation of the constants. The set of quantum numbers $F_{i}, J, N$ identifies a single spectral line by the initial and final states of the transition $\left|N ; b^{1} \Sigma_{g}^{+}\right\rangle \leftarrow\left|F_{i}, J ; X^{3} \Sigma_{g}^{-}\right\rangle$. Primes are here used to distinguish between different spectral lines, rather than different molecular states. Inelastic collisions may couple the initial $(X)$ state $F_{i}, J$ of one spectral line to the initial state $F_{i}^{\prime}, J^{\prime}$ of another line, and the same holds for the final $(b)$ states $N$ and $N^{\prime}$. The corresponding off-diagonal elements of the matrix $\mathbf{A}$ describe line-mixing. The populations in the ground state are given by

$$
P_{F_{i}, J} \equiv \frac{(2 J+1)}{Z} e^{-h c \tilde{\nu}_{F_{i}, J}^{(X)} \bar{\beta}},
$$

with ground state energies $h c \tilde{\nu}_{F_{i}, J}^{(X)}$, obtained from diagonalization of the molecular Hamiltonian [17] with spectroscopic constants given in Table I.

The elements of matrix A in Eq. (13) are given by

$$
\mathrm{A}_{F_{i}^{\prime}, J^{\prime}, N^{\prime} ; F_{i}, J, N}(\tilde{\nu})=\left[\tilde{\nu}-\tilde{\nu}_{F_{i}, J, N}\right] \delta_{J, J^{\prime}} \delta_{F_{i}, F_{i}^{\prime}} \delta_{N, N^{\prime}}-\frac{i n}{2 \pi c}\left\langle v \sigma_{F_{i}^{\prime}, J^{\prime}, N^{\prime} ; F_{i}, J, N}\right\rangle,
$$

with $i$ the imaginary number, $n$ the number density (particles per unit volume) of the perturbers, $c$ the speed of light, $\tilde{\nu}_{F_{i}, J, N}$ transition wavenumbers, and $i n\left\langle v \sigma_{F_{i}^{\prime}, J^{\prime}, N^{\prime} ; F_{i}, J, N}\right\rangle / 2 \pi c$ the relaxation matrix elements $[22,26]$ describing the response of the gas in thermal equilibrium. The latter quantity includes a thermal average over collision energies $E_{c}$ according to

$$
\left\langle v \sigma_{F_{i}^{\prime}, J^{\prime}, N^{\prime} ; F_{i}, J, N}\right\rangle=\left(\frac{8 k_{B} T}{\mu \pi}\right)^{1 / 2}\left(\frac{1}{k_{B} T}\right)^{2} \int_{0}^{\infty} E_{c}\left[\sigma_{F_{i}^{\prime}, J^{\prime}, N^{\prime} ; F_{i}, J, N}^{+}\left(E_{c}\right)+\sigma_{F_{i}^{\prime}, J^{\prime}, N^{\prime} ; F_{i}, J, N}^{-}\left(E_{c}\right)\right] e^{-E_{c} \bar{\beta}} \mathrm{d} E_{c}
$$

in unit volume per unit time, with $v$ denoting the thermal velocity. The cross sections are 
given by

$$
\begin{aligned}
& \sigma_{F_{i}^{\prime}, J^{\prime}, N^{\prime} ; F_{i}, J, N}^{ \pm}\left(E_{c}\right)=\frac{\pi}{2 \mu E_{c}} \sum_{J_{\text {tot }}^{(\mathrm{b})}, J_{\text {tot }}^{(\mathrm{X})}, l_{ \pm}^{\prime}, l_{ \pm}}(-1)^{J-J^{\prime}} \sqrt{\frac{2 J^{\prime}+1}{2 J+1}} \\
& \times\left(2 J_{\text {tot }}^{(\mathrm{b})}+1\right)\left(2 J_{\text {tot }}^{(\mathrm{X})}+1\right)\left\{\begin{array}{ccc}
J & 1 & N \\
J_{\text {tot }}^{(\mathrm{b})} & l_{ \pm} & J_{\text {tot }}^{(\mathrm{X})}
\end{array}\right\}\left\{\begin{array}{ccc}
J^{\prime} & 1 & N^{\prime} \\
J_{\text {tot }}^{(\mathrm{b})} & l_{ \pm}^{\prime} & J_{\text {tot }}^{(\mathrm{X})}
\end{array}\right\} \\
& \times\left[\delta_{F_{i}, F_{i}^{\prime}} \delta_{J, J^{\prime}} \delta_{N, N^{\prime}} \delta_{l_{ \pm}, l_{ \pm}^{\prime}}-S_{N^{\prime}, l_{ \pm}^{\prime} ; N, l_{ \pm}}^{J_{\mathrm{tb}}^{(\mathrm{b})^{*}}}\left(E_{\mathrm{c}}\right) S_{F_{i}^{\prime}, J^{\prime}, l_{ \pm}^{\prime} ; F_{i}, J, l_{ \pm}}^{J_{\mathrm{t}}^{(\mathrm{X})}}\left(E_{\mathrm{c}}\right)\right] .
\end{aligned}
$$

Here $\mu$ is the reduced mass of the perturber-absorber complex, $l_{+}$and $l_{-}$are even and odd partial wave quantum numbers, and $J_{\text {tot }}^{(\mathrm{X})}$ and $J_{\text {tot }}^{(\mathrm{b})}$ are the total angular momentum quantum numbers of the collisional complexes with the $X$ and $b$ electronic states of oxygen. The expressions in curly brackets are six- $j$ symbols. Note that the phase factor $(-1)^{l_{ \pm}-l_{ \pm}^{\prime}}$ is omitted [25], since it is equal to 1 . The scattering matrix elements $S_{\ldots}^{\cdots}\left(E_{\mathrm{c}}\right)$ contain the dynamical information of the binary collisions and are found by solving the coupled-channels equations [17] on a single potential (for either the $\mathrm{He}-\mathrm{O}_{2}\left(X^{3} \Sigma_{g}^{-}\right)$or the $\mathrm{He}_{-} \mathrm{O}_{2}\left(b^{1} \Sigma_{g}^{+}\right)$complex). $S$ matrix elements for both potentials should be calculated at exactly the same collision energy $E_{\mathrm{c}}$. The relation between the total energy $E_{\text {tot }}$ of the scattering calculation and the collision energy is given by

$$
E_{\text {tot }}=E_{\mathrm{c}}+E_{\text {target }}
$$

with $E_{\text {target }}$ the initial-state energy for either $\mathrm{He}-\mathrm{O}_{2}\left(X^{3} \Sigma_{g}^{-}\right)$ground-state or He- $\mathrm{O}_{2}\left(b^{1} \Sigma_{g}^{+}\right)$ excited-state collisions.

Note that the incoming $l$ and outgoing $l^{\prime}$ partial waves are the same for both oxygen states, this is a consequence of the assumption that the absorbing molecule is in an isotropic gas $[22,23]$. Solutions of the coupled-channels equations for our problem have a well defined parity of $(-1)^{N+l+1}$ for the ground state and $(-1)^{N+l}$ for the $b$ state, which allows a separate scattering calculation for odd and even parity. Consequently, this is also possible for Eq. (17). This is the reason for the distinction between even $l_{+}$and odd $l_{-}$partial wave quantum numbers.

In conclusion, the spectrum that follows from Eq. (13) obtains a non-Lorentzian shape if spectral lines are coupled to each other by inelastic collisions, represented by non-zero off-diagonal relaxation matrix elements [Eqs. (15) and (16)]. If, however, spectral lines are 
not coupled to each other, Eq. (13) becomes a sum of Lorentzian line shapes

$$
F(\tilde{\nu})=\frac{1}{\pi} \frac{8 \pi^{3} \tilde{\nu}}{\left(4 \pi \varepsilon_{0}\right) 3 h c^{3}} \sum_{F_{i}, J, N} \frac{\left|\left\langle F_{i}, J ;^{3} \Sigma_{g}^{-}|| \mu \| N ;^{1} \Sigma_{g}^{+}\right\rangle\right|^{2} P_{F_{i}, J}}{\left(\tilde{\nu}-\tilde{\nu}_{F_{i}, J, N}+n d_{F_{i}, J, N}\right)^{2}+\left(n w_{F_{i}, J, N}\right)^{2}} .
$$

The half width at half height $w_{F_{i}, J, N}$ and shift $d_{F_{i}, J, N}$ per unit density of the perturbers of these lines are equal to the real and imaginary parts of $\left\langle v \sigma_{F_{i}, J, N ; F_{i}, J, N}\right\rangle / 2 \pi c$ in Eq. (15).

\section{HE-O ${ }_{2}\left(b^{1} \Sigma_{g}^{+}\right)$INTERACTION POTENTIAL}

In order to perform the scattering calculations which describe the collision dynamics, we require accurate interaction potentials for the $\mathrm{He}-\mathrm{O}_{2}$ complex. For the $\mathrm{He}-\mathrm{O}_{2}\left(X^{3} \Sigma_{g}^{-}\right)$ system, such a potential is available from the literature [15]. This ground state potential was calculated with the partially spin-restricted open-shell single and double excitation coupled cluster method [29] with perturbative triples [30] $[\mathrm{RCCSD}(\mathrm{T})]$. For the $\mathrm{He}_{2} \mathrm{O}_{2}\left(b^{1} \Sigma_{g}^{+}\right)$system, however, we have constructed a new potential based on high-level ab initio calculations, as described below.

\section{A. Ab initio methods}

The He- $\mathrm{O}_{2}\left(b^{1} \Sigma_{g}^{+}\right)$potential was calculated using the MOLPRO [31] package. The coordinate system used for the He- $\mathrm{O}_{2}$ system is defined by the vector $\boldsymbol{R}$ that connects the center of mass of the oxygen molecule to the helium atom; $R$ is the length of this vector and $\theta$ is the angle between this vector and the $\mathrm{O}_{2}$ bond axis $\boldsymbol{r}$. The O-O distance $r$ is fixed. The basis set used consists of an augmented correlation consistent triple zeta (aug-cc-pVTZ) basis [32-34] on the $\mathrm{He}$ and $\mathrm{O}$ atoms, and an additional set of (3s3p2d1f) bond functions defined by Tao and Pan [35]. For each $\mathrm{He}_{2} \mathrm{O}_{2}$ geometry these bond functions are centered on the intersection of the vector $\boldsymbol{R}$ and the ellipse that is chosen such that it passes through the midpoint of $R$ in the T-shaped geometry and through the midpoint of the smallest $\mathrm{O}$-He distance in the linear geometry, see Ref. 15. We apply a correction for the basis set superposition error with the Boys and Bernardi counterpoise procedure [36].

The RCCSD(T) method used previously for the ground state potential [15] cannot be used for the $b$ state, since two states of $A^{\prime}$ symmetry (point group $\mathrm{C}_{s}$ ) are involved that 
correlate with the ${ }^{1} \Sigma_{g}^{+}$and ${ }^{1} \Delta_{g}$ states of the free oxygen molecule. To obtain an interaction potential of approximately the $\operatorname{RCCSD}(\mathrm{T})$ quality, a correction was devised as follows.

We used the complete active space self consistent field [37, 38] (CASSCF) program to distinguish the 4 lowest states of oxygen: $X^{3} \Sigma_{g}^{-}, a^{1} \Delta_{g}\left(A^{\prime}\right.$ and $A^{\prime \prime}$ symmetry), and $b^{1} \Sigma_{g}^{+}$. All core orbitals were used in the active space to avoid problems with the convergence of the calculation at small $\mathrm{He}-\mathrm{O}_{2}$ distances. The resulting orbitals of a CASSCF calculation were used as the starting orbital guess for each subsequent geometry, starting at large He$\mathrm{O}_{2}$ distance. The canonical orbitals corresponding to the $\mathrm{He}_{2} \mathrm{O}_{2}\left(a^{1} \Delta_{g}\right)$ and $\mathrm{He}-\mathrm{O}_{2}\left(b^{1} \Sigma_{g}^{+}\right)$ complexes with $A^{\prime}$ symmetry were used as input for a state-averaged complete active space second order perturbation theory [39] (CASPT2) calculation. For every grid point the energy corresponding to the state with the highest reference energy was used for the He- $\mathrm{O}_{2}\left(b^{1} \Sigma_{g}^{+}\right)$ potential. To obtain a potential of approximately $\operatorname{RCCSD}(\mathrm{T})$ quality, the difference in energy between the CASPT2 and RCCSD(T) calculations for $\mathrm{He}_{2} \mathrm{O}_{2}\left(X^{3} \Sigma_{g}^{-}\right)$was subtracted from the CASPT2 $\mathrm{He}-\mathrm{O}_{2}\left(b^{1} \Sigma_{g}^{+}\right)$potential at each geometry.

\section{B. Ab initio grid}

The interaction energy was calculated for 532 geometries. A rotational constant of $B_{0}=1.3912 \mathrm{~cm}^{-1}[28]$ was used to fix the $\mathrm{O}_{2}$ vibrational coordinate $r$ to a value of $r_{0}=2.326 \mathrm{a}_{0}$. The radial grid was spaced with steps of $0.2 \mathrm{a}_{0}$ from $2.9 \mathrm{a}_{0}$ to $8.1 \mathrm{a}_{0}$ and extended logarithmically with $R_{i+1} / R_{i}=1.1$ from $8.5 \mathrm{a}_{0}$ to $20.05 \mathrm{a}_{0}$, with one extra point at $25 \mathrm{a}_{0}$. The angular grid was confined to the range of $0^{\circ}$ to $90^{\circ}$, and consisted of 14 Gauss-Legendre quadrature points.

\section{The fit of the potential}

The interaction potential $V(R, \theta)$ was first expanded in Legendre polynomials of even order,

$$
V(R, \theta)=\sum_{l=0,2, \ldots}^{12} C_{l}(R) P_{l}(\cos \theta) .
$$

The radial coefficients were obtained via a Gauss-Legendre quadrature

$$
C_{l}(R)=\sum_{i=1}^{14}(2 l+1) w_{i} V\left(R, \theta_{i}\right) P_{l}\left(\cos \theta_{i}\right),
$$


with $0 \leq \theta_{i} \leq \frac{\pi}{2}$ and $w_{i}$ the quadrature points and their corresponding weights. After performing the 14-point quadrature of Eq. (21), the ab initio points could be reproduced via Eq. (20) with relative errors on the order of $0.1 \%$ in the repulsive wall at small $R$ and $0.01 \%$ and less near the potential well and at larger $R$.

To obtain a fit of the radial coefficients $C_{l}(R)$, the fit function was split in a long range part $C_{l}^{(\mathrm{lr})}(R)$ and short range part $C_{l}^{(\mathrm{sr})}(R)$

$$
C_{l}(R)=C_{l}^{(\mathrm{sr})}(R)+C_{l}^{(\mathrm{lr})}(R)
$$

The long range part is given by

$$
C_{l}^{(\mathrm{lr})}(R)=-\frac{c_{n l} f_{n}(\zeta R)}{R^{-n}}
$$

where $n=l+4$, except for $l=0$ for which $n=6$. These are the leading terms in the multipole expansion [40]. The $f_{n}$ 's are Tang-Toennies damping functions [41]

$$
f_{n}(x)=1-e^{-x} \sum_{k=0}^{n} \frac{x^{k}}{k !} .
$$

To obtain a reliable fit of the $c_{n l}$ coefficients in Eq. (23), we set the damping functions to 1 and performed a weighted least squares fit of $C_{l}(R)$ for $R \geq 10 \mathrm{a}_{0}$. The weighting function was set to $R^{n}$ with $n$ in accordance with Eq. (23). The first three coefficients are given in Table II. We then chose $\zeta=2.0 \mathrm{a}_{0}^{-1}$ for $C_{l}^{(\mathrm{lr})}(R)$ in Eq. (23) and obtained $C_{l}^{(\mathrm{sr})}(R)$ in Eq. (22), by subtraction of $C_{l}^{(\mathrm{lr})}(R)$ from the ab initio points. For the short range part, the reproducing kernel Hilbert space (RKHS) method with the reproducing kernel for distance-like variables was used $[42,43]$. The RKHS parameter $m$ was set to the leading term parameter $n$ minus 1 and the RKHS smoothness parameter was set to 2 .

After the RKHS fitting, reconstruction of $V(R, \theta)$ via Eqs. (20) and (22) reproduced the $a b$ initio points with a relative error on the order of $0.01 \%$ or better for $R$ larger than $4 \mathrm{a}_{0}$. This was also the case for 35 randomly chosen geometries not used for the fit. In the region of $2.9<R<4.0$ a $_{0}$ the largest relative error is $0.49 \%$. The fit of the ab initio potential behaves unphysically for $R<2.9 \mathrm{a}_{0}$ (interaction energies $\geq 0.05 E_{h} \approx 11,000 \mathrm{~cm}^{-1}$ ). Figure 2 presents contour plots of the newly computed interaction potential for the $b$ state and the potential for the ground state for a comparison. The $b$ state potential in Fig. 2(a) is slightly more anisotropic than the ground state potential of Fig. 2(b). The global minimum for the excited state potential is at the T-shaped geometry at $R=5.9 \mathrm{a}_{0}$ with dissociation energy 
$D_{e}=152.2 \mu E_{h}$, and for the ground state $D_{e}=127.1 \mu E_{h}$ at $R=6.0 \mathrm{a}_{0}$. The local minimum for the excited state at the linear geometry has a well depth of $123.7 \mu E_{h}$ at $R=6.9 \mathrm{a}_{0}$, and for the ground state $116.7 \mu E_{h}$ at $R=6.9 \mathrm{a}_{0}$.

\section{SCATTERING CALCULATIONS}

In order to obtain the $S$ matrix elements needed for Eq. (17) we solved the coupledchannels equations using the renormalized Numerov method [44], with matrix elements in a Hund's case (b) basis as provided in Ref. 17. All scattering code was written in MatLAB [45]. The equations were integrated per parity, see section II C, and per $J_{\text {tot }}$. The solutions were then matched to asymptotic boundary conditions in a basis of channel eigenfunctions. The same code was used for the scattering calculations of helium with both the oxygen $b$ and $X$ state.

To test our scattering code we calculated the inelastic cross sections reported in Ref. 16 for the ground state potential as a function of collision energy. After correction of a small mistake in the code used in Ref. 16 we obtained good agreement for all cross sections.

Both ground and $b$ state $S$ matrix elements were calculated at the same collision energy $E_{\mathrm{c}}$, Eq. (18). A full scattering calculation was thus required per target state, initial or final state of the transition, per collision energy. The total energy [Eq. (18)] determined the basis set size used for the scattering calculations. For the ground state we used the same settings as in [16]: $N \leq 9$ for total energies up to $30 \mathrm{~cm}^{-1}, N \leq 11$ for total energies up to $50 \mathrm{~cm}^{-1}$, $N \leq 13$ for total energies up to $100 \mathrm{~cm}^{-1}, N \leq 15$ for total energies up to $200 \mathrm{~cm}^{-1}, N \leq 17$ for total energies up to $300 \mathrm{~cm}^{-1}, N \leq 21$ for total energies up to $500 \mathrm{~cm}^{-1}, N \leq 23$ for total energies up to $750 \mathrm{~cm}^{-1}, N \leq 27$ for total energies up to $1000 \mathrm{~cm}^{-1}$, and $N \leq 29$ for total energies up to $2500 \mathrm{~cm}^{-1}$. For the $b$ state we used $N \leq 12$ for total energies up to $30 \mathrm{~cm}^{-1}$, $N \leq 14$ for total energies up to $50 \mathrm{~cm}^{-1}, N \leq 16$ for total energies up to $100 \mathrm{~cm}^{-1}, N \leq 18$ for total energies up to $200 \mathrm{~cm}^{-1}, N \leq 22$ for total energies up to $300 \mathrm{~cm}^{-1}, N \leq 24$ for total energies up to $500 \mathrm{~cm}^{-1}, N \leq 26$ for total energies up to $750 \mathrm{~cm}^{-1}, N \leq 30$ for total energies up to $1000 \mathrm{~cm}^{-1}$, and $N \leq 32$ for total energies up to $2500 \mathrm{~cm}^{-1}$.

The step size for the propagation was set to 10 steps per de Broglie wavelength and the maximum propagation radius was set to $20 \mathrm{a}_{0}$ for collision energies $\leq 600 \mathrm{~cm}^{-1}, 18 \mathrm{a}_{0}$ for $\leq 1600 \mathrm{~cm}^{-1}$, and $16 \mathrm{a}_{0}$ for $>1600 \mathrm{~cm}^{-1}$. The propagation radii were determined by 
visual inspection of the convergence of the diagonal $S$ matrix elements as a function of the propagation radius for a range in $J_{\text {tot }}$ 's and a selection of total energies. This criterion is used since $S$ matrix elements for both potentials should be of the same quality. The convergence criterion was a change of less than $10^{-4}$ in the absolute value of the complex number and of less than 3 degrees for its phase. The highest target state incorporated in the calculations of the spectrum are for the oxygen ground state $J=16$, and for the $b$ state $N=16$.

The convergence criterion for $J_{\text {tot }}$ during the scattering calculations, was that the average absolute value of the transition matrix elements should be smaller than $10^{-4}$ for two values of $J_{\text {tot }}$ separated by a preset step size. The convergence criterion is sensitive to the magnitude of the $S$ matrix elements. Since a product of $S$ matrix elements from both potentials is required in Eq. (17), this criterion is a direct measure for the significance of the contribution from the $S$ matrix element to the cross section. The $J_{\text {tot }}$ step size for the ground state scattering was set to 5 and for the $b$ state to 6 starting from $J_{\text {tot }}=0$. Typical maximum values of $J_{\text {tot }}$ were 20 for scattering energies on the order of $10 \mathrm{~cm}^{-1}$, and 150 for scattering energies above $1600 \mathrm{~cm}^{-1}$, depending on the target state. After determining the maximum $J_{\text {tot }}$ we performed the calculations for the values of $J_{\text {tot }}$ that were skipped in first instance.

The spectrum [Eq. (13] requires a thermal average over collision energies as given in Eq. (16). For this averaging a grid of collision energies was used that started at 1 in steps of 0.5 up to $50 \mathrm{~cm}^{-1}$, from 50 in steps of 5 to $200 \mathrm{~cm}^{-1}$, from 200 in steps of 25 to $1000 \mathrm{~cm}^{-1}$, and from 1000 in steps of 100 to $1900 \mathrm{~cm}^{-1}$. This grid was used for a trapezoidal integration to obtain the thermally averaged relaxation matrix elements. The total number of scattering calculations was nearly 12,000 .

\section{CAVITY RING-DOWN EXPERIMENTS}

\section{A. The experimental setup}

Cavity ring-down experiments were carried out on two $\mathrm{O}_{2}$-helium mixtures $(14.65 \%$ and $\left.1 \% \mathrm{O}_{2}\right)$. The experiments on the $1 \%$ mixture were performed on the setup used in [46] and illustrated in Fig. 3. For the $14.65 \%$ the experiments were carried out on the setup used in [6]. The main difference between these setups is the pulsed laser source, which is a diode laser (Toptica DL100 with a power of about $50 \mathrm{~mW}$ and a wavelength $770 \pm 20 \mathrm{~nm}$ ) in 
the first case and a dye (pyridine-2) laser (Scanmate from Lambda Physik, Ft. Lauterdale, FL) pumped with a Nd:YAG (neodymium:yttrium aluminium garnet) in the second. The former has an effective bandwidth of $0.016 \mathrm{~cm}^{-1}$ and the latter $0.07 \mathrm{~cm}^{-1}$. In the remainder of this section, the cavity ring-down experiments will be discussed for the diode laser setup illustrated in Fig. 3, with the understanding that it is essentially the same as the dye laser setup.

An optical cavity was constructed inside a pressure cell using two mirrors with a reflectivity above $99.99 \%$ (Research electro optics) that are separated by approximately $350 \mathrm{~mm}$, resulting in an effective absorption path length of $3.5 \mathrm{~km}$. The mirrors have a radius of curvature of $1000 \mathrm{~mm}$. Each measurement commenced with emptying our pressure cell to a pressure of a few millibar using a membrane pump (Pfeifer Vacuum MVP 055-3). Subsequently, we slowly filled the pressure cell with a mixture of $14.65 \% \mathrm{O}_{2}$ (Linde gas, 99.9999\% purity) and $85.35 \%$ He (Linde gas, 99.999\% purity) using two flow controllers (Bronkhorst High-Tech). For the $1 \%$ mixture an in-house premixed bottle containing $1.00 \pm 0.01 \% \mathrm{O}_{2}$ in helium was used. During measurements, the wavelength is kept fixed at a position where the contribution of spectral lines of the molecular oxygen isotopologues is expected to be negligible. We continuously measured the pressure inside the cell with a diaphragm pressure detector (Pfeiffer Vacuum D-35614), accuracy 0.3\%. We detected the light leaking out of the cavity with an avalanche photodiode (APD, Licel GmbH LP-1A series). When the intensity behind the cavity reaches a threshold of approximately $40 \%$ of the maximum possible intensity in the cavity, the laser was disabled within $1 \mu \mathrm{s}$ for a period of approximately $200 \mu \mathrm{s}$ by a home-built electronic switch. We simultaneously recorded the ring-down signal, the pressure, and the laser current with an oscilloscope (HP infinium). All experiments where performed at a temperature of $293 \pm 1 \mathrm{~K}$.

\section{B. Data processing}

\section{Removal of CIA and Rayleigh scattering}

The ring-down signals as a function of time $t$ and threshold laser intensity $I_{0}(\tilde{\nu})$ were fitted by an exponent of the form [6]

$$
I(t)=I_{0}(\tilde{\nu}) e^{-\frac{t}{\tau(\tilde{\nu})}}
$$


with $\tau(\tilde{\nu})$ the characteristic ring-down time given by

$$
\tau(\tilde{\nu})=\frac{d}{c} \frac{1}{|\ln (\mathcal{R})|+\kappa(\tilde{\nu}) d} .
$$

Here $d$ is the cavity length, $c$ the speed of light, $\mathcal{R}$ the reflectivity of the mirrors and $\kappa(\tilde{\nu})$ the extinction coefficient. As already mentioned in the introduction, the extinction coefficient is understood to consist of contributions from: Rayleigh scattering [47], collision induced absorption (CIA) [3], and magnetic dipole absorption, that are summed as [6]

$$
\kappa(\tilde{\nu})=\sigma(\tilde{\nu}) n_{\mathrm{O}_{2}}+c(\tilde{\nu}) n_{\mathrm{O}_{2}}^{2}+\alpha(\tilde{\nu})
$$

Here $n_{\mathrm{O}_{2}}$ is the number density of molecular oxygen in amagat, and the terms with $\sigma(\tilde{\nu})$ and $c(\tilde{\nu})$ are due to Rayleigh scattering and CIA, respectively. The magnitude of the He and $\mathrm{O}_{2}$ Rayleigh scattering coefficients $\sigma(\tilde{\nu})$ is well known. Due to the low atomic polarizability of helium, its Rayleigh scattering is negligible. Also the CIA due to helium-oxygen collisions can be neglected, based on yet unpublished data measured with our cavity ring-down setup. Therefore, the magnetic dipole absorption $\alpha(\tilde{\nu})$ could be obtained from Eq. (27) by subtracting only the Rayleigh scattering and CIA contributions for oxygen. For the index of refraction of $\mathrm{O}_{2}$ and the King correction factor (molecular anisotropy) [48] we used the paper by Bates [49]. The $\mathrm{O}_{2}-\mathrm{O}_{2}$ CIA contribution was obtained from the data by Tran et al. [8]. Their data was interpolated using a cubic spline, and has been validated by independent measurements within our group [6,9]. The uncertainty in this contribution is about $10 \%$, however it contributes little to the absorption strength as Fig. 1 shows and does not cause a systematic error. The coefficients $\sigma(\tilde{\nu})$ and $c(\tilde{\nu})$ used in Eq. (27) are given in Table IV.

The observed absorption, which scales with the square of the pressure, is dominated by the magnetic dipole transition, and the observed line width is mainly due to the effects of $\mathrm{O}_{2}$-He collisions. This will be illustrated in Sec. VIB, where we directly compare the data extracted from the measurements with calculated pressure broadening coefficients, and in Sec. VIC, where it will be shown that collisional line broadening is by far the strongest effect.

\section{Refining the $14.65 \% \mathrm{O}_{2}$ data}

As will be shown in the results section, the bandwidth of the dye laser used to perform the measurements on the $14.65 \% \mathrm{O}_{2}$-helium mixture is broad enough to excite multiple 
cavity modes. This leads to multiple pressure dependent absorption curves with similar curvature, but with different offsets at zero pressure due to the wavelength dependence of the mirror reflectivity. Using the assumption that thermal fluctuations in the setup are the cause of the scatter in the data belonging to a single curve, we attempted to separate the data points belonging to curves with different offsets. This is in order to make the curvature more pronounced for a comparison with the theoretical predictions that should reproduce the curvature.

To this end, the pressure dependent data sets were filtered keeping in mind that the resulting data set should reveal a normally distributed scatter. The focus was on obtaining the absorption curve with the lowest offset, so that the filter could be kept simple. A 20point-window was moved over the data point-by-point while each time collecting a number of points with the lowest absorption. The number of points was chosen per data set such that the residuals between the filtered data set and a low order polynomial fit through this data resembles a normal distribution. A $2 \sigma$ standard deviation was subsequently determined from these residuals, and used to remove the scarce outliers on the low absorption side. The final step was to set the offset to zero for the filtered data set by subtraction of the zero-order term in the fitted polynomial.

\section{RESULTS AND DISCUSSION}

In this section our theoretical results are compared to experimental results taken from the literature and from measurements performed on our cavity ring-down setup. First we present integrated line cross sections and pressure broadening coefficients, both quantities that are relatively insensitive to the effects of line-mixing, hence line-mixing is neglected in the calculations. Then the effect of the line-mixing mechanism is studied by the prediction of pressure-dependent absolute absorption strengths in the "valleys" between spectral lines. As will be shown, the difference between including and neglecting the line-mixing becomes most pronounced in these regions. When the line-mixing is neglected, the spectrum is a sum of Lorentzian line shapes according to Eq. (19). The results of the calculations are compared to the cavity ring-down data.

For the discussion we designate the spectral lines of transition type $\left|N^{\prime} M_{N}^{\prime} ; b^{1} \Sigma_{g}^{+}\right\rangle$ $\leftarrow\left|F_{i} J M_{J} ; X^{3} \Sigma_{g}^{-}\right\rangle$(section II B) by ${ }^{\Delta N} \Delta J(N)$. Here $N$ is the nuclear rotation quantum 
number in Eq. (2), depending on the choice for $F_{i}$ as the initial state of the magnetic dipole transition. Furthermore, $\Delta N \equiv N^{\prime}-N$ and $\Delta J \equiv N^{\prime}-J$, with $\Delta X=-1,0,1$ denoted by $P, Q, R$, respectively. Hence designations such as ${ }^{P} Q(3)$ or ${ }^{R} R(3)$.

\section{A. Integrated line cross sections}

Table III shows a comparison between our integrated line cross sections calculated with Eq. (10), and experimentally determined cross sections [50] for the $P$ branch $(P$ is related to $\Delta N)$. These integrated cross sections test the magnetic dipole transition matrix elements for molecular oxygen, excluding any collisional effects. The experiments were performed in a pressure range from 1.4 to $4.6 \mathrm{kPa}(\approx 0.013$ to $0.045 \mathrm{~atm})$ on a $2 \%$ oxygen mixture with nitrogen. The partition sum $Z=215.77$ (at a temperature of $296 \mathrm{~K}$ ) and the transition wavenumbers $\tilde{\nu}_{F_{i}, J, N}$ of Eq. (10) were taken from the literature $[20,51]$ based on data from the Hitran database. Relative differences with respect to the experimental values are given, and show an agreement to within $1 \%$. This confirms that the value of $0.0268 \mu_{B}$ from Ref. 11 that we used for the electronic magnetic dipole transition moment is indeed accurate.

\section{B. Pressure broadening and shifting coefficients}

The first test for collisional effects is the prediction of helium- $\mathrm{O}_{2}$ broadening and shifting coefficients of the spectral lines in the A-band spectrum. These coefficients represent the linear dependence of the line widths and shifts on the, in this case, helium density. The broadening coefficients have been determined experimentally, and are reported in the literature [52]. The line shift coefficients, however, were too small to determine accurately. The effect of line-mixing on the line widths is expected to be negligible, since there is no significant overlap between spectral lines at the relatively low pressures for which the experiments were carried out $\left(0.26 \mathrm{~atm} \mathrm{O}_{2}\right.$ partial pressure and a range of 0.13 to $0.52 \mathrm{~atm}$ helium pressure). If line-mixing is neglected the spectrum becomes a sum of Lorentz lines as given in Eq. (19). Using Eqs. (15) and (16) the pressure broadening and shifting coefficients can be defined. We define the coefficients $\gamma_{k}$ for the $k$-th spectral line in units of $\mathrm{cm}^{-1} \mathrm{~atm}^{-1}$ for comparison with the experiment ( $k$ corresponds to a set of quantum numbers $N^{\prime}, F_{i}, J$ 
of the transition)

$$
\gamma_{k} \equiv \frac{1}{2 \pi c} \frac{1}{p_{0}} \frac{T_{0}}{T} N_{L}\left\langle v \sigma_{k ; k}\right\rangle .
$$

Here $p_{0}=1 \mathrm{~atm}, T_{0}=273.15 \mathrm{~K}, N_{L}$ is Loschmidt's number $\left(2.6867774 \cdot 10^{25}\right.$ particles $\mathrm{m}^{-3}$ for an ideal gas at $0^{\circ} \mathrm{C}$ and $\left.1 \mathrm{~atm}\right)$, and $\left\langle v \sigma_{k ; k}\right\rangle$ is $\left\langle v \sigma_{F_{i}, J, N ; F_{i}, J, N}\right\rangle$ of Eq. (16). The real and imaginary part of $\gamma_{k}$ represent the broadening and shifting coefficients of spectral line $k$.

Figure 4 shows that the calculated broadening coefficients follow a smooth line and capture the trend and magnitude of the experimentally determined coefficients. The apparent structure in the experimental points of Fig. 4 is not reproduced, but it is not obvious that this structure is significant. One argument in favor of our calculated trend, is that the measured oxygen self-broadening coefficients, indicated with the open black dots, display the same trend (by an overall factor of almost $4 / 3$ ). The reported uncertainties for these coefficients [53] are on the order $10^{-4} \mathrm{~cm}^{-1} \mathrm{~atm}^{-1}$ and are not visualized. These self-broadening coefficients will be used for the calculation of the absolute absorption strengths in section VIC.

The pressure shifts that follow from the imaginary part of Eq. (28) are shown in Fig. 5. All shift coefficients have the same sign, and are indeed smaller than what could experimentally be determined by the spectrometer used by [52]. It should be noted that the present theory does not exactly satisfy detailed balance. This is known to affect the imaginary part of the relaxation matrix in particular $[54,55]$.

\section{Pressure-dependent absorption strengths}

The results shown so far, prove that the strong absorption features of the calculated and experimental spectrum are in good agreement. The next step is to study the weak features of the spectrum, that are most sensitive to the effects of line-mixing. A comparison between theory and experiment will put the line-mixing incorporated in the current theory to the test. One tests the formalism, the interaction helium- $\mathrm{O}_{2}$ interaction potentials, and especially the off-diagonal elements in the relaxation matrix.

For this comparison one should look at the spectrum to find out where line-mixing becomes most apparent. Hereto, we calculated a part of the A-band spectrum using Eq. (13). The transition wavenumbers $\tilde{\nu}_{F_{i}, J, N}$ for the spectrum, required in Eq. (10), were taken from the literature $[20,51]$ and are taken from the Hitran database. Since the collisional effects 
on the experimental spectrum are not solely from the helium- $\mathrm{O}_{2}$ collisions, but also from $\mathrm{O}_{2}-\mathrm{O}_{2}$ collisions, the relaxation matrix elements in Eq. (15) should be amended to include $\mathrm{O}_{2}$ self-broadening and shifting. The corrected matrix elements, proportional to the helium density $n_{\mathrm{He}}$ in amagat, are

$$
n\left\langle v \sigma_{k^{\prime} ; k}^{\mathrm{He}-\mathrm{O}_{2}, \mathrm{O}_{2}-\mathrm{O}_{2}}\right\rangle \equiv 2 \pi c p_{0} \frac{T}{T_{0}} n_{\mathrm{He}}\left(\gamma_{k^{\prime}, k}^{\mathrm{He}-\mathrm{O}_{2}}+\frac{x_{\mathrm{O}_{2}}}{1-x_{\mathrm{O}_{2}}} \gamma_{k}^{\mathrm{O}_{2}-\mathrm{O}_{2}} \delta_{k^{\prime}, k}\right)
$$

with

$$
\gamma_{k^{\prime}, k}^{\mathrm{He}-\mathrm{O}_{2}} \equiv \frac{1}{2 \pi c} \frac{1}{p_{0}} \frac{T_{0}}{T} N_{L}\left\langle v \sigma_{k^{\prime} ; k}\right\rangle,
$$

with $\left\langle v \sigma_{k^{\prime} ; k}\right\rangle$ the $a b$ initio helium- $\mathrm{O}_{2}$ matrix elements of Eqs. (15) and (16) including offdiagonal elements. The real part of $\gamma_{k}^{\mathrm{O}_{2}-\mathrm{O}_{2}}$ is the experimentally determined oxygen selfbroadening coefficient (shown in Fig. 4) and the imaginary part the shift for the $k$-th spectral line taken from the literature [53]. These coefficients only contribute to the relaxation matrix diagonal, which is indicated by the Kronecker delta $\delta_{k^{\prime}, k}$. The $x_{\mathrm{O}_{2}}$ is the fraction of oxygen in the gas mixture used in the experiment. The calculated spectrum is shown as the black line in Fig. 6(a). This figure provides the cross sections of oxygen molecules surrounded by helium at a pressure of $5 \mathrm{~atm}$ to accentuate the collisional effects. The fraction $x_{\mathrm{O}_{2}}$ was set to zero so only the collisional effects of helium are visible.

In Fig. 6 we demonstrate the effect of line-mixing by also plotting the ratio of this spectrum divided by the spectrum without line-mixing, Eq. (19), as a solid gray line. The ratios 1.0 and 0.7 are indicated as dashed horizontal gray lines. One observes that the effect of line-mixing is strongest in between the lines. Clearly, the strongest effect is found near $13122 \mathrm{~cm}^{-1}$ in between the $P$ branch $\left(\tilde{\nu}<13120 \mathrm{~cm}^{-1}\right)$ and the $R$ branch $(\tilde{\nu}>13128$ $\mathrm{cm}^{-1}$ ), where line-mixing reduces the absorption cross section. In other minima line-mixing increases the absorption cross section.

Figure 6(a) contains five arrows indicating the spectral positions where the pressuredependent cavity ring-down measurements were performed. Each arrow is labeled by a letter corresponding to a panel below. The fraction of oxygen in the gas mixture is indicated in the upper left corner of each panel. Each gray dot in the figure corresponds to one measured ring-down curve. As was discussed in section $\mathrm{V}$, each ring-down curve is fitted to obtain the extinction coefficient $\kappa(\tilde{\nu})$ at a given pressure. There are between 1000 and 3000 points per panel. 
The contributions of CIA and Rayleigh scattering were subtracted from the measured pressure dependent curves, as described in section VB 1. The values of the coefficients used for Eq. (27) are given in Table IV. What remains after these corrections is the magnetic dipole absorption strength $\alpha(\tilde{\nu})$. The experimental data in Fig. 6 is shown as a function of the helium density $n_{\mathrm{He}}$. The panels displaying the data for the $14.65 \% \mathrm{O}_{2}$ mixture contain dark gray data points that indicate what part of the gray data points belong to one cavity mode, as was discussed in section V B 2. Figure 6(b) provides an example where two absorption curves are visible with different offsets but similar curvature.

Each panel contains a solid line with the absolute predictions of the absorption strength including line-mixing. To link the spectrum $F(\tilde{\nu})$ to the absorption strength we used the relation

$$
\alpha(\tilde{\nu})=x_{\mathrm{O}_{2}} \frac{T_{0}}{T} \frac{p}{p_{0}} N_{L} F(\tilde{\nu}),
$$

where the pressure $p$ is directly measured in the cavity ring-down experiment. Note that Eq. (29) is used in Eq. (13) to compute $F(\tilde{\nu})$. The dashed line ignores the line-mixing, the off-diagonal elements of the relaxation matrix, and corresponds to a spectrum built as a sum of Lorentzian line shapes. We also compare our data to a semi-empirical model developed by Tonkov et al. $[12,13]$, shown as a dash-dotted line in Fig. 6. This model is based on the "strong collision" approximation, in which it is assumed that the probability to find the active molecule in specific final $J$ states after a collision is given by the thermal distribution function and does not depend on its initial $J$ value. Then, the only parameters needed to construct the effect of line-mixing due to the off-diagonal terms in the relaxation matrix of Eq. (15) are related to the diagonal elements of this matrix: the (measured) line frequencies and relative intensities and the collision frequency. The latter can be derived from the observed line broadening parameters. The Tonkov model was developed for vibrational transitions; in Refs. 56, 57 it was applied to electronic transitions. It is empirical but highly predictive.

It can be seen that our $a b$ initio results in which line-mixing effects are included are in good quantitative agreement with the experimental data for all wavelengths considered. This demonstrates the validity of our quantum mechanical framework. In a number of cases the difference between the present purely $a b$ initio and the empirical model is very small. Figure 6(c) is taken in the minimum of the spectrum of Fig. 6(a), where the effect of line-mixing is largest. Here the experiment agrees with the two models that include line-mixing, while the 
neglect of line mixing leads to a $30 \%$ deviation. The difference between our first principles calculation and the empirical model is not sufficiently large to allow an experimental test. The fact that we find such good agreement with the empirical data puts the Tonkov model on a more solid footing.

\section{CONCLUSIONS}

The pressure broadening of the rotationally resolved oxygen A-band spectrum by helium was studied theoretically. To our knowledge this is the first time pressure broadening of an electronic transition of a molecule is treated with a fully quantum mechanical description. A new interaction potential for helium $-\mathrm{O}_{2}\left(b^{1} \Sigma_{g}^{+}\right)$was computed. This potential and a helium- $\mathrm{O}_{2}\left(X^{3} \Sigma_{g}^{-}\right)$potential taken from the literature were required for the scattering calculations to calculate line broadening in the impact approximation. Regarding the spectroscopy of molecular oxygen, accurate integrated line cross sections for 13 spectral lines in the $P$ branch were obtained. The line broadening coefficients predicted by the impact approximation treatment are in good agreement with experimental values from the literature. From cavity ring-down experiments absolute absorption strengths were obtained between several spectral lines, with a sensitivity of about $10^{-7} \mathrm{~cm}^{-1}$. The experimental results are in quantitative agreement with those of the calculations. In particular in the region between the $P$ and $R$ branch it is proven that neglect of the off-diagonal elements in the relaxation matrix responsible for line-mixing, would lead to a $30 \%$ mismatch with the experimentally determined absorption strengths. Additionally it was shown that the $a b$ initio calculated line-mixing is in quantitative agreement with the empirical Tonkov model.

To put the present work in a more general perspective, let us reiterate that collisional effects on line shapes are small but relevant. Especially for our Earth's atmosphere an $a b$ initio treatment of both line mixing and CIA due to collisions with oxygen and nitrogen is of enormous value, as it will allow predictions of the effects of the large differences in temperatures and other atmospheric conditions. This theoretical and experimental study of the $\mathrm{O}_{2}$-He system constitutes the first successful step. Both line broadening and line mixing are well described, even though these effects are small and near the limit of what can be measured by the most sensitive techniques available to date. For $\mathrm{O}_{2}-\mathrm{N}_{2}$ and $\mathrm{O}_{2}-\mathrm{O}_{2}$ collisions the effects are expected to be substantially larger. The success of the Tonkov model, which 
uses measured line broadening coefficients to predict line mixing, stresses the common origin of both processes: atom-molecule collisions. It will be interesting to see whether also in more complex collision systems the results of the Tonkov model will still be similar to ab initio predictions. This treatment of line mixing is part of an ambitious project which aims at theoretically predicting CIA in electronic transitions. Such predictions do not exist yet, while experiments show that in this case collisions between oxygen and between oxygen and other molecules have very different and ill understood effects. It was found, for example, that CIA spectra due to oxygen-oxygen collisions are much stronger than for oxygen-nitrogen collisions and also have a very different shape as a function of wavelength. Moreover, the relative effect of collisions with oxygen and nitrogen is very different for excitation to the $a^{1} \Delta_{g}$ state than for excitation to the $b^{1} \Sigma_{g}^{+}$state.

\section{Acknowledgments}

The authors thank Leo Meerts for his aid and suggestions. Francois Lique is thanked for his aid in tracking the source of the difference between the results of his and our scattering calculations. Leander Gerritsen is thanked for preparation of the gas mixtures used in the experiments.

[1] B. v. Diedenhoven, O. P. Hasekamp, and I. Aben, Atmos. Chem. Phys. 5, 2109 (2005).

[2] B. v. Diedenhoven, O. P. Hasekamp, and J. Landgraf, J. Geophys. Res. 112, D15208 (2007).

[3] L. Frommhold, Collision-Induced Absorption in Gases, Cambridge Univ. Press, Cambridge, 1994.

[4] B. F. Minaev and H. Ågren, J. Chem. Soc., Faraday Trans. 93, 2231 (1997).

[5] B. F. Minaev and G. I. Kobzev, Spectrochim. Acta A 59, 3387 (2003).

[6] F. R. Spiering, M. B. Kiseleva, N. N. Filippov, H. Naus, B. van Lieshout, C. Weijenborg, and W. J. van der Zande, J. Chem. Phys. 133, 114305 (2010).

[7] A. Predoi-Cross, K. Harnbrook, R. Keller, C. Povey, I. Schofield, D. Hurtmans, H. Over, and G. C. Mellau, J. Mol. Spectrosc. 248, 85 (2008).

[8] H. Tran, C. Boulet, and J.-M. Hartmann, J. Geophys. Res. 111, 15210 (2006). 
[9] D. A. Long, D. J. Robichaud, and J. T. Hodges, J. Chem. Phys. 137, 014307 (2012).

[10] J. H. v. Vleck, Astrophys. J. 80, 161 (1934).

[11] B. Minaev, O. Vahtras, and H. Ågren, Chem. Phys. 208, 299 (1996).

[12] M. O. Bulanin, A. B. Dokuchaev, M. V. Tonkov, and N. N. Filippov, J. Quant. Spectrosc. Radiat. Transfer 31, 521 (1984).

[13] M. V. Tonkov, N. N. Filippov, Y. M. Timofeyev, and A. V. Polyakov, J. Quant. Spectrosc. Radiat. Transfer 56, 783 (1996).

[14] J.-M. Hartmann, C. Boulet, and D. Robert, Collisional effects on molecular spectra, Elsevier, 2008.

[15] G. C. Groenenboom and I. M. Struniewicz, J. Chem. Phys. 113, 9562 (2000).

[16] F. Lique, J. Chem. Phys. 132, 044311 (2010).

[17] G. C. Corey and F. R. McCourt, J. Phys. Chem. 87, 2723 (1983).

[18] L. C. Biedenharn and J. D. Louck, Angular Momentum in Quantum Physics, volume 8 of Encyclopedia of Mathematics, Addison-Wesley, Reading, 1981.

[19] P. R. Bunker and P. Jensen, Molecular Symmetry and Spectroscopy, NRC Research Press, Ottawa, second edition, 1998.

[20] J. Fischer, R. R. Gamache, A. Goldman, L. Rothman, and A. Perrin, J. Quant. Spectrosc. Radiat. Transfer 82, 401 (2003).

[21] R. Klotz, C. M. Marian, S. D. Peyerimhoff, B. A. Hess, and R. J. Buenker, Chem. Phys. 89, 223 (1984).

[22] A. Ben-Reuven, Phys. Rev. 141, 34 (1966).

[23] A. Ben-Reuven, Phys. Rev. 145, 7 (1966).

[24] R. Shafer and R. G. Gordon, J. Chem. Phys. 58, 5422 (1973).

[25] G. C. Corey, J. Chem. Phys. 81, 2678 (1984).

[26] U. Fano, Phys. Rev. 131, 259 (1963).

[27] Y. Endo and M. Mizushima, Jpn. J. Appl. Phys. 21, 1379 (1982).

[28] P. H. Krupenie, J. Phys. Chem. Ref. Data 1, 423 (1972).

[29] P. J. Knowles, C. Hampel, and H.-J. Werner, J. Chem. Phys. 112, E3106 (2000).

[30] J. D. Watts, J. Gauss, and R. J. Bartlett, J. Chem. Phys. 98, 8718 (1993).

[31] H.-J. Werner, P. J. Knowles, and et al., MOLPRO: a package of ab initio programs, version 2010.1. 
[32] T. H. Dunning, J. Chem. Phys. 90, 1007 (1989).

[33] R. A. Kendall, T. H. Dunning, and R. J. Harrison, J. Chem. Phys. 96, 6796 (1992).

[34] D. E. Woon and T. H. Dunning, J. Chem. Phys. 100, 2975 (1994).

[35] F. M. Tao and Y. K. Pan, J. Chem. Phys. 97, 4989 (1992).

[36] S. F. Boys and F. Bernardi, Mol. Phys. 19, 553 (1970).

[37] P. J. Knowles and H.-J. Werner, Chem. Phys. Lett. 115, 259 (1985).

[38] H.-J. Werner and P. J. Knowles, J. Chem. Phys. 82, 5053 (1985).

[39] H.-J. Werner, Mol. Phys. 89, 645 (1996).

[40] A. van der Avoird, P. E. S. Wormer, F. Mulder, and R. M. Berns, Top. Curr. Chem. 93, 1 (1980).

[41] K. T. Tang and J. P. Toennies, J. Chem. Phys. 80, 3726 (1984).

[42] T.-S. Ho and H. Rabitz, J. Chem. Phys. 104, 2584 (1996).

[43] T.-S. Ho and H. Rabitz, J. Chem. Phys. 113, 3960 (2000).

[44] B. R. Johnson, NRCC Proceedings 5, 86 (1979).

[45] Matlab, The MathWorks, Inc., http://www.mathworks.com.

[46] F. R. Spiering and W. J. van der Zande, Phys. Chem. Chem. Phys. 14, 9923 (2012).

[47] J. W. Strutt, Philosophical Magazine 47, 375 (1899).

[48] L. V. King, Proceedings of the Royal Society of London. Series A 104, 333 (1923).

[49] D. R. Bates, Planet Space Sci. 32, 785 (1984).

[50] D. J. Robichaud, J. T. Hodges, L. R. Brown, D. Lisak, P. Masłowski, L. Y. Yeung, M. Okumura, and C. E. Miller, J. Mol. Spectrosc. 248, 1 (2008).

[51] L. Rothman et al., J. Quant. Spectrosc. Radiat. Transfer 110, 533 (2009).

[52] R. S. Pope, P. J. Wolf, and G. P. Perram, J. Mol. Spectrosc. 223, 205 (2004).

[53] L. R. Brown and C. Plymate, J. Mol. Spectrosc. 199, 166 (2000).

[54] J. Boissoles, C. Boulet, and X. Bruet, J. Chem. Phys. 116, 7537 (2002).

[55] L. Monchick, J. Chem. Phys. 95, 5047 (1991).

[56] F. R. Spiering, M. B. Kiseleva, N. N. Filippov, L. van Kesteren, and W. J. van der Zande, Phys. Chem. Chem. Phys. 13, 9616 (2011).

[57] F. R. Spiering, M. B. Kiseleva, N. N. Filippov, H. Naus, B. Lieshout, A. M. van der Veen, and W. J. van der Zande, Mol. Phys. 109, 535 (2011). 
TABLE I: Spectroscopic constants of $\mathrm{O}_{2}$ in $\mathrm{cm}^{-1}$ used in this work. $B_{0}$ and $D_{0}$ are the rotational and distortion constant of the vibrational ground state, and $\lambda_{S S}$ and $\lambda_{N S}$ the spin-spin and spinrotation coupling constants.

\begin{tabular}{ccc}
\hline \hline constant & $X^{3} \Sigma_{g}^{-}[27]$ & $b^{1} \Sigma_{g}^{+}[28]$ \\
\hline$B_{0}$ & 1.437675 & 1.391247 \\
$D_{0}$ & $4.790 \cdot 10^{-6}$ & $5.375 \cdot 10^{-6}$ \\
$\lambda_{S S}$ & 1.984751 & - \\
$\lambda_{N S}$ & $-8.425 \cdot 10^{-3}$ & - \\
\hline \hline
\end{tabular}


TABLE II: Fitted leading term coefficients of Eq. (23) in $E_{h} / a_{0}^{n}$.

\begin{tabular}{llr}
\hline \hline$l$ & $n$ & $c_{n, l}$ \\
\hline 0 & 6 & 10.26 \\
2 & 6 & 2.44 \\
4 & 8 & -3.19 \\
\hline \hline
\end{tabular}


TABLE III: Comparison of experimental [50] and calculated [Eq. (10], $T=296 \mathrm{~K}$ ) integrated line cross section in units of $10^{-24} \mathrm{~cm}$ molecule ${ }^{-1}$. Numbers in parentheses are $1 \sigma$ standard deviations in the unit of the last digit. The relative difference with respect to the experimental values is given as a percentage.

\begin{tabular}{lcllllll}
\hline \hline line & ${ }^{P} Q(3)$ & ${ }^{P} P(3)$ & ${ }^{P} Q(5)$ & ${ }^{P} P(5)$ & ${ }^{P} Q(7)$ & ${ }^{P} P(7)$ & ${ }^{P} Q(9)$ \\
\hline$[50]$ & $3.901(8)$ & $5.738(8)$ & $5.974(9)$ & $7.557(9)$ & $7.098(9)$ & $8.423(10)$ & $7.256(8)$ \\
calculated & 3.887 & 5.717 & 5.966 & 7.558 & 7.093 & 8.400 & 7.263 \\
rel. diff. \% & -0.35 & -0.37 & -0.13 & 0.01 & -0.07 & -0.27 & 0.10 \\
\hline line & ${ }^{P} P(9)$ & ${ }^{P} Q(11)$ & ${ }^{P} P(11)$ & ${ }^{P} Q(13)$ & ${ }^{P} P(13)$ & ${ }^{P} Q(15)$ & \\
\hline$[50]$ & $8.262(8)$ & $6.667(7)$ & $7.437(7)$ & $5.608(5)$ & $6.113(5)$ & $4.338(4)$ & \\
calculated & 8.276 & 6.661 & 7.402 & 5.575 & 6.088 & 4.303 & \\
rel. diff. \% & 0.17 & -0.09 & -0.47 & -0.59 & -0.40 & -0.81 & \\
\hline \hline
\end{tabular}


TABLE IV: Coefficients $c(\tilde{\nu})$ and $\sigma(\tilde{\nu})$ used in Eq. (27) to subtract the contributions of CIA and Rayleigh scattering, respectively, from the measured pressure dependent extinction $\kappa(\tilde{\nu})$ at wavenumber $\tilde{\nu}$.

\begin{tabular}{ccc}
\hline \hline$\tilde{\nu}$ & $c(\tilde{\nu})$ & $\sigma(\tilde{\nu})$ \\
$\mathrm{cm}^{-1}$ & $10^{-7} \mathrm{~cm}^{-1}$ amagat $^{-2}$ & $10^{-8} \mathrm{~cm}^{-1}$ amagat $^{-1}$ \\
\hline 13081.30 & 1.90 & 2.87 \\
13103.13 & 1.79 & 2.89 \\
13112.48 & 1.76 & 2.89 \\
13122.48 & 2.00 & 2.90 \\
13125.92 & 1.98 & 2.91 \\
\hline \hline
\end{tabular}




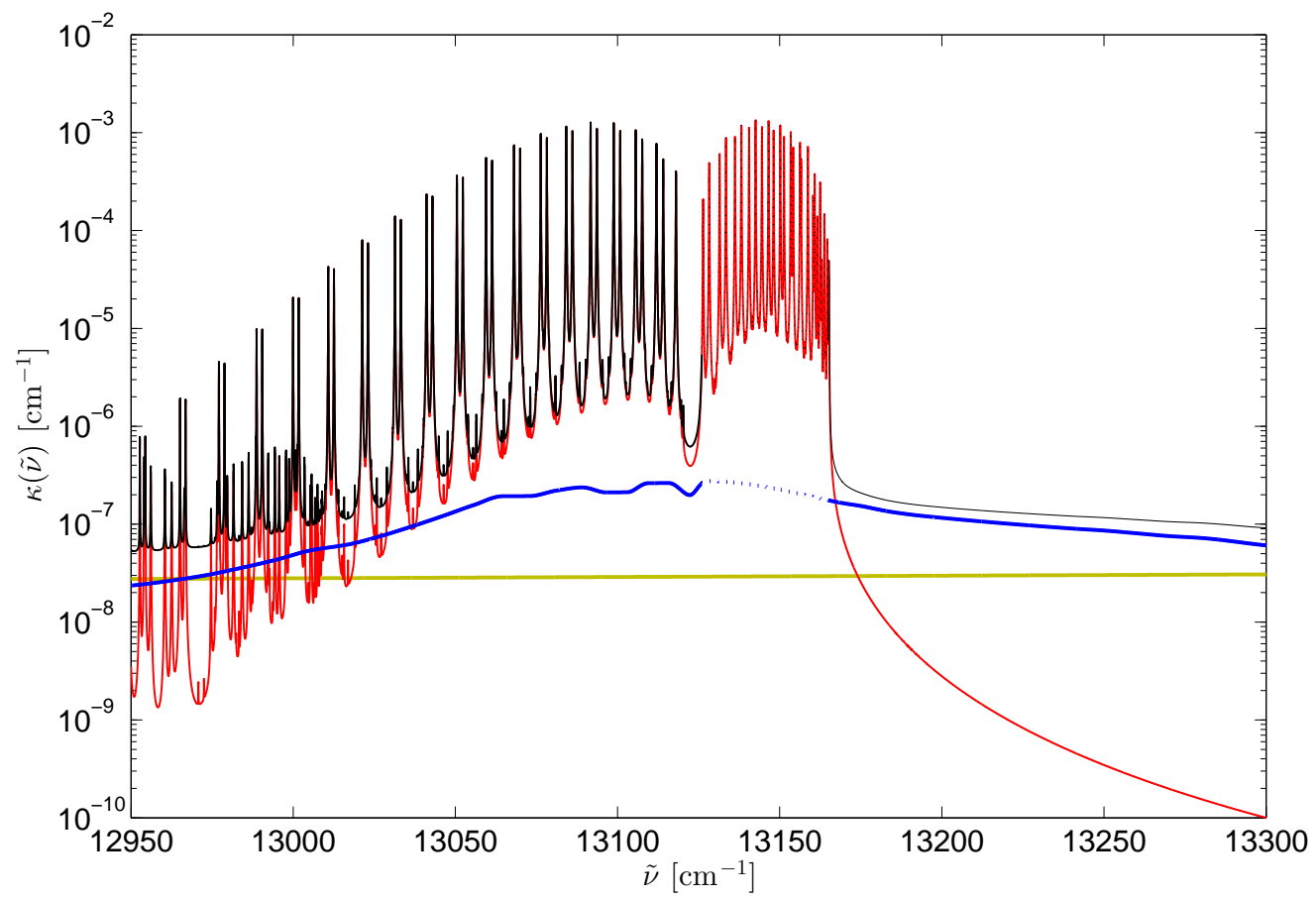

FIG. 1: Construction of the extinction coefficient $\kappa(\tilde{\nu})$ as a function of wavenumber $\tilde{\nu}$ for molecular oxygen gas (black) at a density of 1 amagat in the A-band region from the summed contributions of: Rayleigh scattering (green), magnetic dipole absorption (red), and CIA (blue). The dashes in the region of the $R$ branch indicate that the CIA is not determined here. This figure was reprinted with permission from J. Chem. Phys. 133, 114305 (2010) [6]. Copyright 2010 American Institute of Physics. 


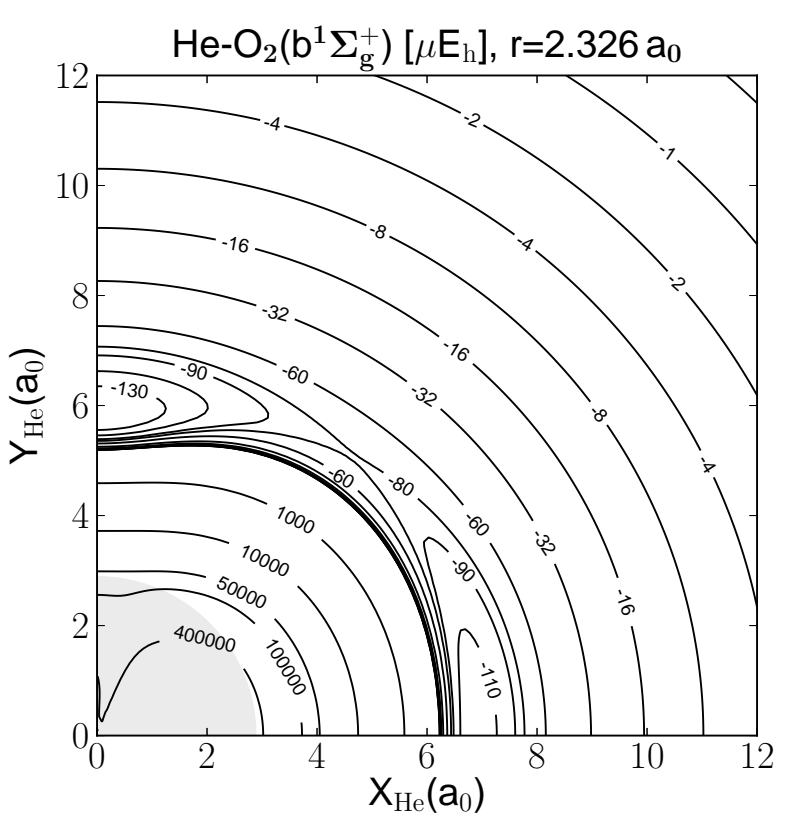

(a)

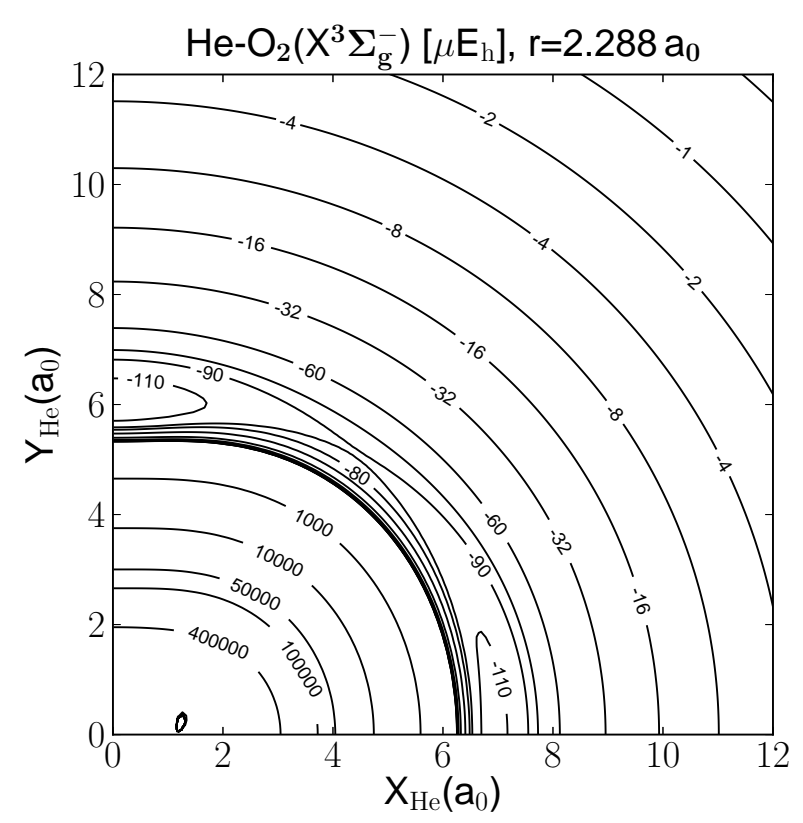

(b)

FIG. 2: The two potential surfaces, in $\mu E_{h}$, used in the scattering calculations, with the $\mathrm{O}-\mathrm{O}$ distance $r$ indicated in the titles. The molecular axis of the oxygen molecule is parallel to the $\mathrm{X}_{\mathrm{He}}$ axis. (a) The computed He- $\mathrm{O}_{2}\left(b^{1} \Sigma_{g}^{+}\right)$interaction potential, unphysical region indicated in gray. (b) The He- $\mathrm{O}_{2}\left(X^{3} \Sigma_{g}^{-}\right)$interaction potential from [15]. 


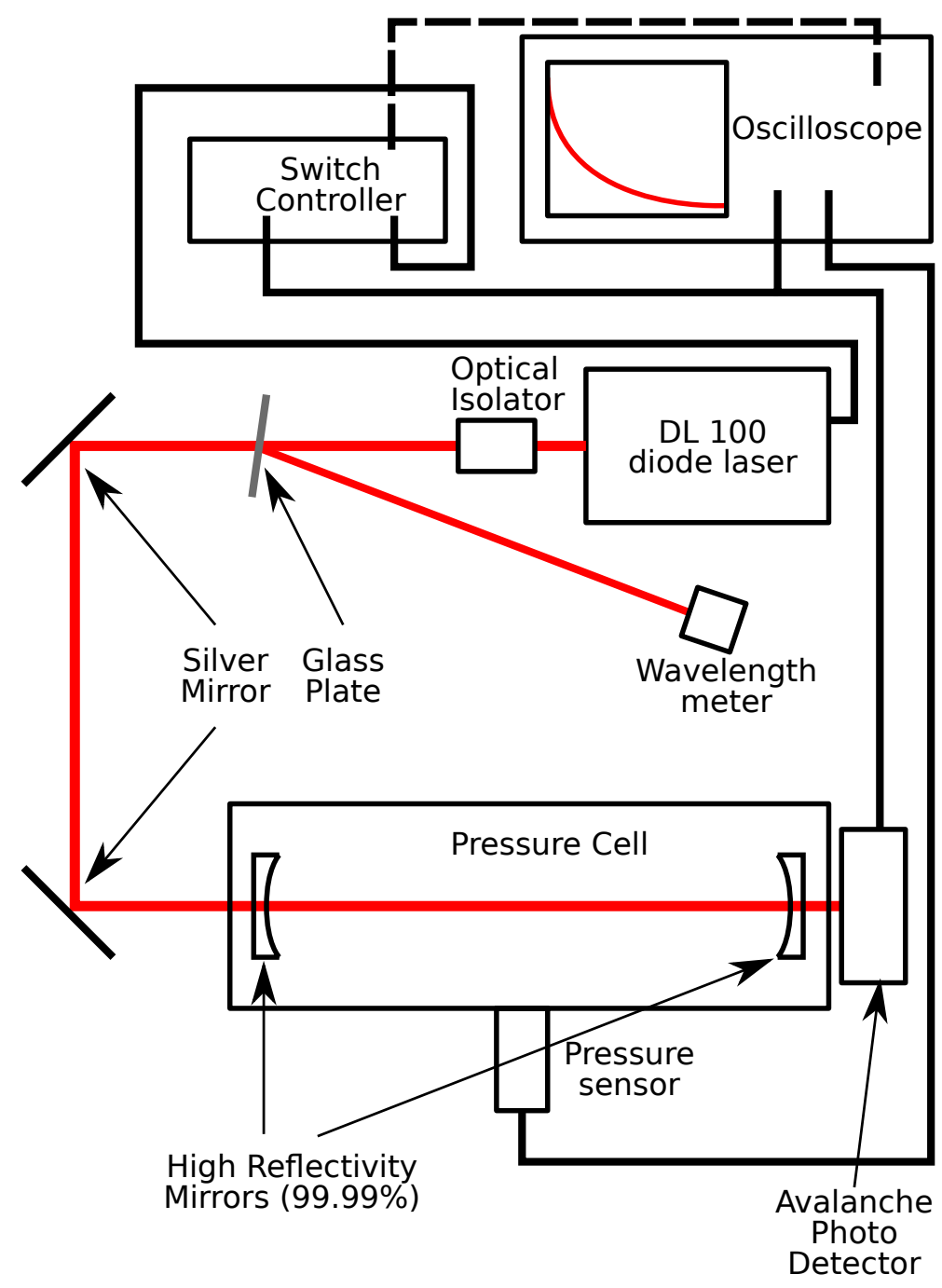

FIG. 3: The cavity ring-down setup used for the determination of pressure dependent extinction. Light from the DL100 laser diode is coupled into the optical ring-down cavity, and is partly used for the determination of the wavelength. The light leaking out of the cavity is detected using an avalanche photo diode. When the detected signal reaches a threshold, the switch controller switches the laser off and provides a trigger for the oscilloscope. The oscilloscope captures the exponentially decaying signal detected by the avalanche photo diode as well as the pressure measured by the pressure sensor. 


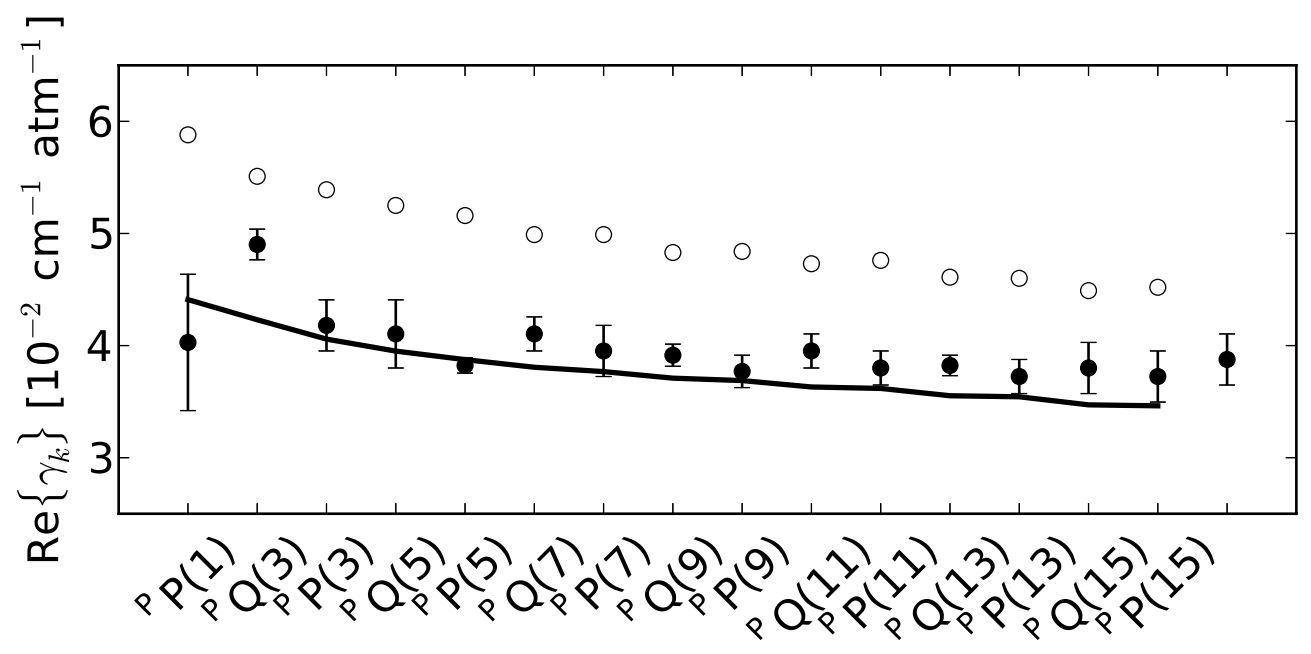

(a) $P$ branch pressure broadening coefficients

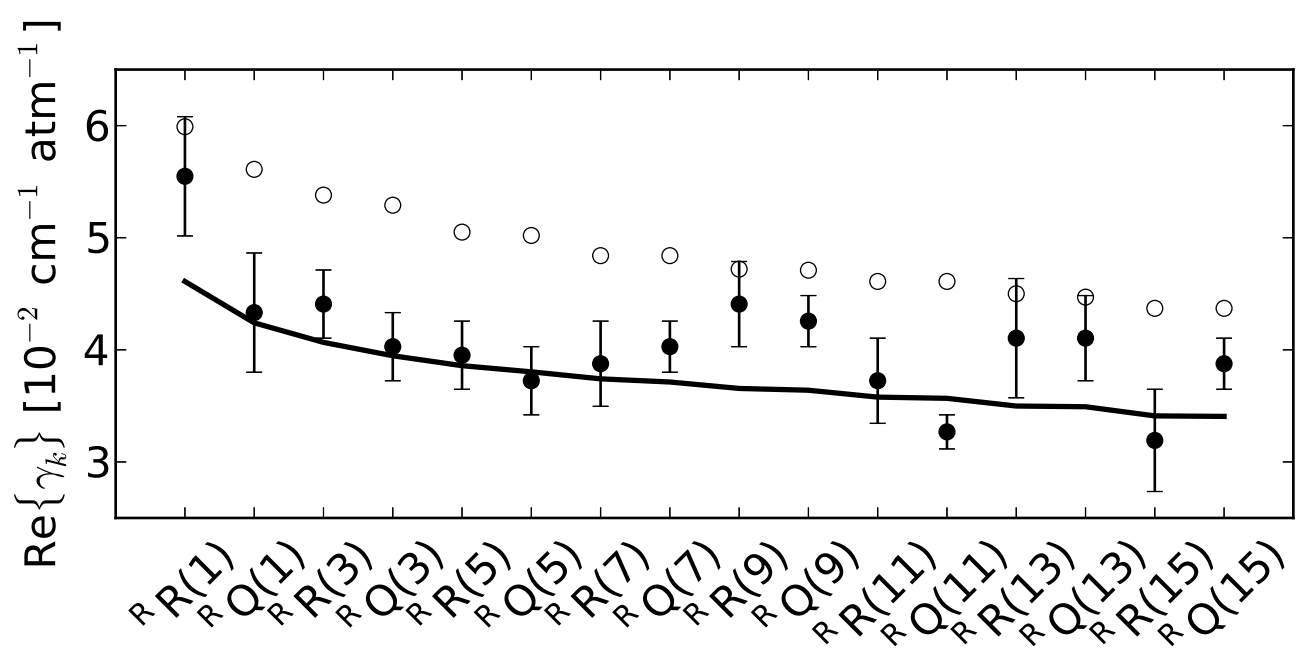

(b) $R$ branch pressure broadening coefficients

FIG. 4: Comparison between experimentally determined line broadening coefficients for helium$\mathrm{O}_{2}[52]$ (solid black dots with $1 \sigma$ error bars), and theory (the real part of Eq. 28) (black line), with $T=298 \mathrm{~K}$ for the $P$ and $R$ branches of the spectrum. The open black dots are experimentally determined oxygen self-broadening coefficients [53]. These are used in the calculations of the absorption strengths in section VIC. Uncertainties for these coefficients [53] are on the order $10^{-4}$ $\mathrm{cm}^{-1} \mathrm{~atm}^{-1}$ and are not visualized. 


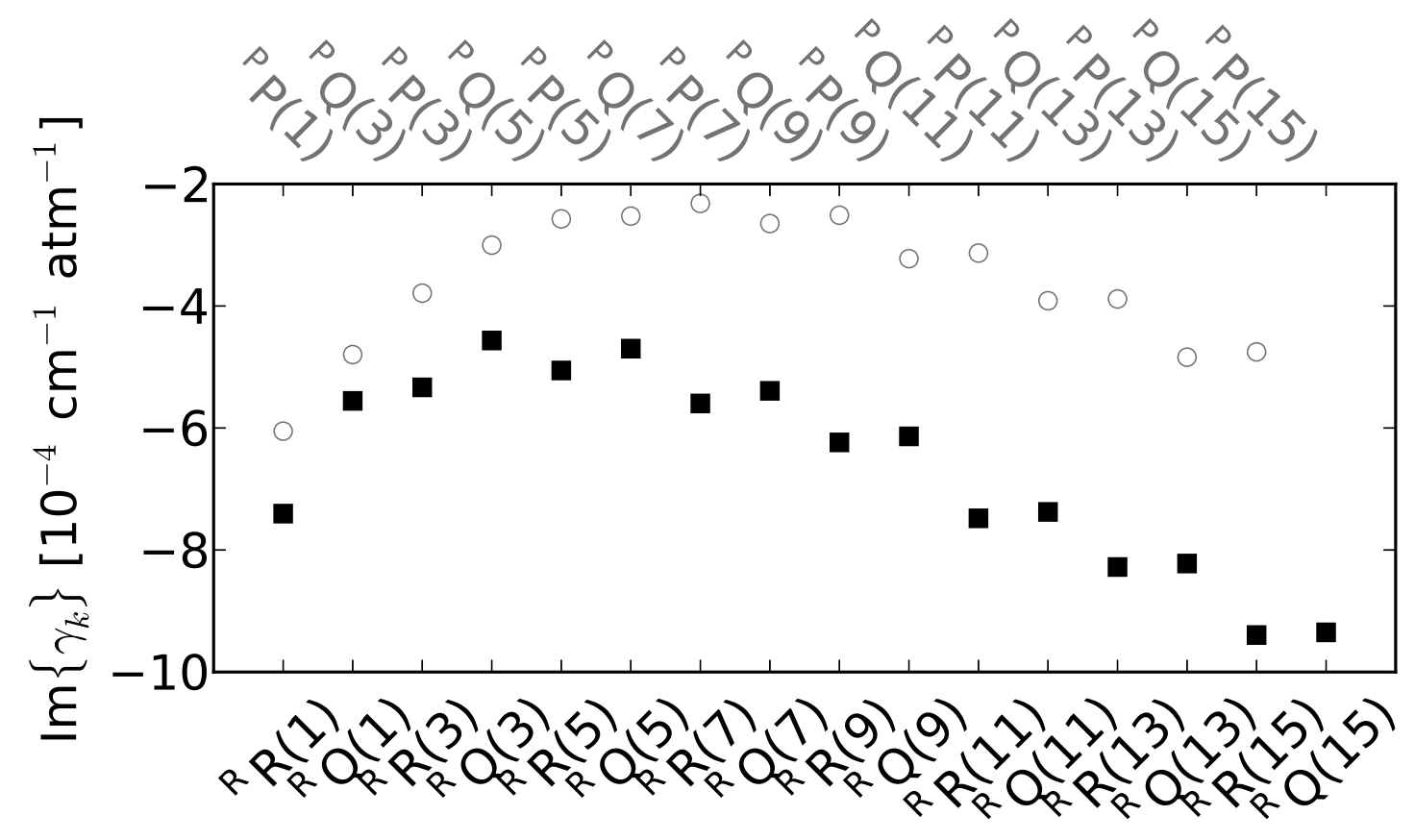

FIG. 5: Calculated pressure shift coefficients, the imaginary part of Eq. (28) at $T=298 \mathrm{~K}$. Coefficients for the $P$ and $R$ branch are indicated in gray open dots and black solid squares, respectively. 

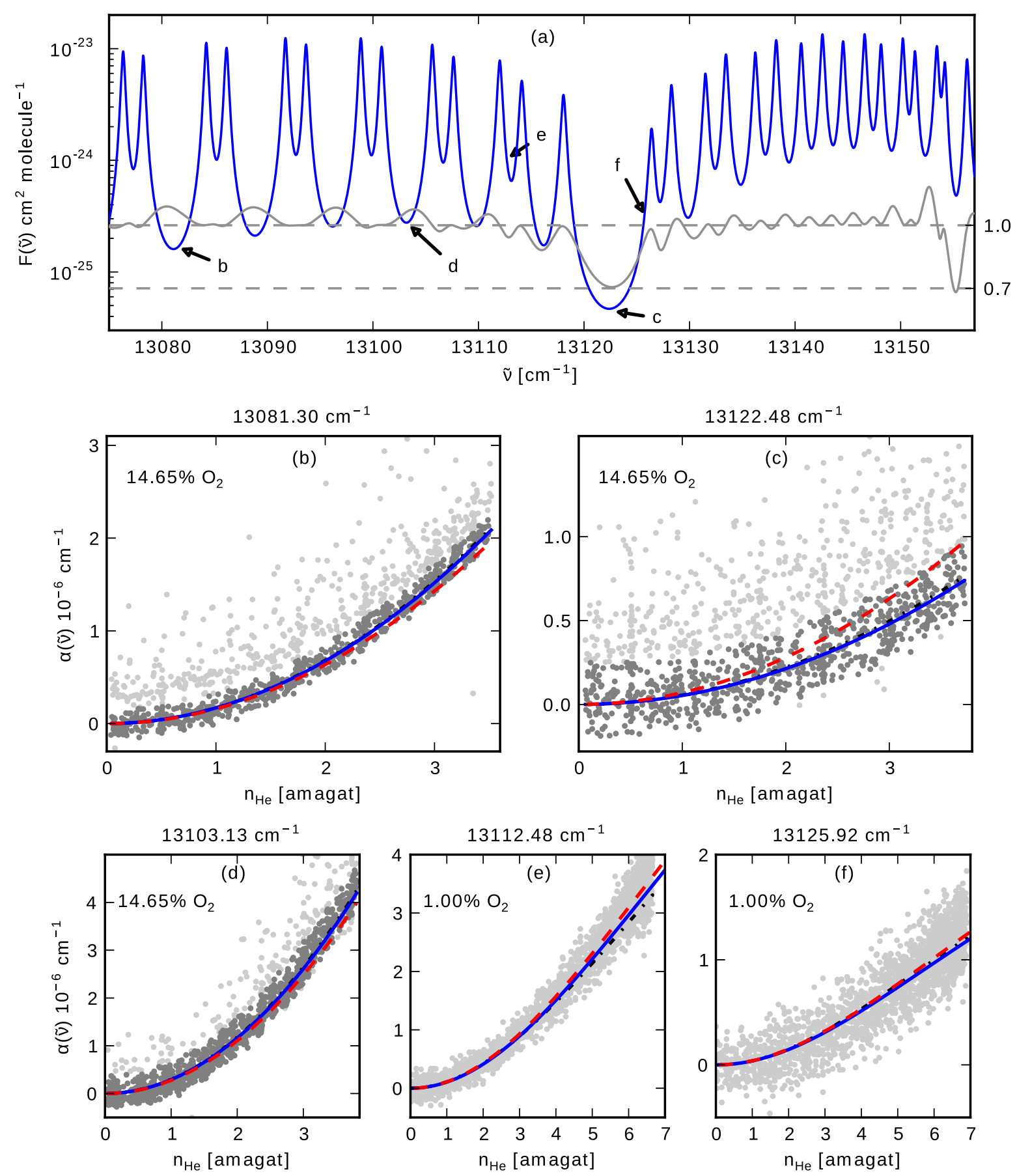

FIG. 6: (a) The calculated absorption spectrum (blue), including line-mixing [Eq. (13)], on a logarithmic intensity scale at $T=293 \mathrm{~K}$ and a partial helium pressure of $5 \mathrm{~atm}$. The solid gray line represents the division of this spectrum by the spectrum without line-mixing [Eq. (19)]. The dashed gray lines indicate the ratios of 1.0 and 0.7. The arrows indicate the spectral position for the experimental data in the corresponding panel. (b-f) Measured magnetic dipole absorption strength $\alpha(\tilde{\nu})$ (gray points) as a function of helium density $n_{\mathrm{He}}$. Dark gray points indicate data belonging to one cavity-mode. Other lines are line-mixing (solid blue), no line-mixing (dashed red) and the Tonkov model (dash-dotted black). 